\title{
Protostellar Feedback Halts the Growth of the First Stars in the Universe
}

\author{
Takashi Hosokawa ${ }^{1,2}$, Kazuyuki Omukai ${ }^{2}$, Naoki Yoshida ${ }^{3}$, and Harold W. Yorke ${ }^{1}$ \\ ${ }^{1}$ Jet Propulsion Laboratory, California Institute of Technology, Pasadena CA 91109, USA \\ ${ }^{2}$ Department of Physics, Kyoto University, Kyoto 606-8502, Japan \\ ${ }^{3}$ Institute for the Physics and Mathematics of the Universe, Todai Institutes for Advanced Study, \\ University of Tokyo, Kashiwa, Chiba 277-8568, Japan
}

*To whom correspondence should be addressed; E-mail: hosokwtk@gmail.com

\begin{abstract}
The first stars fundamentally transformed the early universe by emitting the first light and by producing the first heavy elements. These effects were predetermined by the mass distribution of the first stars, which is thought to have been fixed by a complex interplay of gas accretion and protostellar radiation. We performed radiation-hydrodynamics simulations that followed the growth of a primordial protostar through to the early stages as a star with thermo-nuclear burning. The circumstellar accretion disk was evaporated by ultraviolet radiation from the star when its mass was 43 times that of the Sun. Such massive primordial stars, in contrast to the often postulated extremely massive stars, may help explain the fact that there are no signatures of the pair-instability supernovae in abundance patterns of metal-poor stars in our galaxy.
\end{abstract}

Theoretical studies and detailed computer simulations show that the cradles of the first stars were dense concentrations of primordial gas, with masses of $\sim 1000$ that of the sun. Such gas clouds formed through radiative cooling, with hydrogen molecules at the center of a dark matter halo of $10^{6}$ solar mass $\left(M_{\odot}\right)$, when the age of the universe was a few hundred million years old (1).

According to our current understanding of star formation, a gas cloud's dense core gravitationally contracts in a non-homologous run-away fashion, in which the densest parts become denser faster than does the rest of the cloud (2-5). In a primordial gas cloud, one or a few embryo protostars are formed near the center $(2,6)$. The initial mass of these embryo protostars is only $\simeq 0.01 M_{\odot}$; the bulk of the dense core material remains in the surrounding envelope and is subsequently drawn toward the protostar (or protostars) through gravity. With the typical angular momentum of dense cores, the centrifugal barrier allows only a small amount of infalling gas to accrete directly onto the star. Instead, a circumstellar disk is formed and the gas is accreted onto the central star through the disk (7). The final mass of these first stars is fixed, when the mass accretion terminates. However, when and how this termination occurs are largely unknown.

Because the luminosity increases rapidly with protostellar mass, radiative feedback is expected to regulate the mass accretion and ultimately shut off the accretion flow, setting the final mass of the first stars. A primordial star more massive than a few tens times the mass of the Sun radiates a copious amount of hydrogen ionizing photons $(>13.6 \mathrm{eV})$. As an accreting star grows in mass, the ionized region in its vicinity grows, and eventually the circumstellar disk is directly exposed to the stellar ionizing radiation. The gas on the disk surface is photoionized and heated and evaporates away from the star-disk system. A 
semi-analytical model of this process predicts that this photoevaporation quenches the accretion flow of the disk material and puts an end to the stellar growth (8). However, the interplay between the accretion flow and the stellar radiation is highly dynamical and complex.

To identify the exact mechanism that halts the growth of a first star and to determine its final mass, we applied a method used for studying the present-day massive star formation $(9,10)$ to the case of the formation of the first stars in a proper cosmological context. We followed the radiation hydrodynamic evolution in the vicinity of an accreting protostar, incorporating thermal and chemical processes in the primordial gas in a direct manner. We also followed the evolution of the central protostar selfconsistently by solving the detailed structure of the stellar interior with zero metallicity as well as the accretion flow near the stellar surface [supporting online material (SOM) text] (11,12). We configured the initial conditions by using the results of a three-dimensional (3D) cosmological simulation, which followed the entire history from primeval density fluctuations to the birth of a small seed protostar at the cosmological redshift $14(6)$. Specifically, when the maximum particle number density reached $10^{6} \mathrm{~cm}^{-3}$ in the cosmological simulation, we considered a gravitationally bound sphere of radius $0.3 \mathrm{pc}$ around the density peak, which enclosed a total gas mass of $\simeq 300 M_{\odot}$. We reduced the 3D data to an axisymmetric structure by averaging over azimuthal angles.

The system was evolved until the central particle number density reached $10^{12} \mathrm{~cm}^{-3}$. We then introduced a sink cell of size $\simeq 10$ astronomical units $(\mathrm{AU})$ and followed the subsequent evolution of the central protostar with a stellar evolution code. The accretion rate onto the protostar was calculated directly from the mass influx through the sink-cell boundary, whereas the luminosity from the protostar, which controls the radiative feedback, was calculated consistently from the protostellar model by using the derived accretion rate.

At its birth, a very small protostar of $\sim 0.01 M_{\odot}$ was surrounded by a molecular gas envelope of $\sim 1 M_{\odot}$, which was quickly accreted onto the protostar. Atomic gas further out initially had too much angular momentum to be accreted directly, and a circumstellar disk formed. The infalling atomic gas first hit the disk plane roughly vertically at supersonic velocities. A shock front formed; behind the shock, the gas cooled and settled onto the disk, and its hydrogen was converted to the molecular form via rapid gas phase three-body reactions. The molecular disk extended out to $\simeq 400 \mathrm{AU}$ from the protostar, when the stellar mass was $10 M_{\odot}$. Accretion onto the protostar proceeded through this molecular disk as angular momentum was transported outward. The accretion rate onto the protostar was $\simeq 1.6 \times 10^{-3} M_{\odot}$ year $^{-1}$ at that moment.

The evolution of the central protostar is determined by competition between mass growth by accretion and radiative energy loss from the stellar interior. The time scale for the former is the accretion timescale $t_{\text {acc }} \equiv M_{*} / \dot{M}$, where $M_{*}$ is mass of the protostar and $\dot{M}$ is mass accretion rate, whereas that for the latter is the Kelvin-Helmholtz $(\mathrm{KH})$ timescale $t_{\mathrm{KH}} \equiv G M_{*}^{2} / R_{*} L_{*}$, where $L_{*}$ is the luminosity from the stellar interior, $R_{*}$ is radius of the star, and $G$ is gravitational constant. The total luminosity of the protostar $L_{\text {tot }}$ is the sum of the stellar luminosity $L_{*}$ and accretion luminosity $L_{\text {acc }} \equiv G M_{*} \dot{M} / R_{*}(13)$.

In the early accretion phase, the stellar radius remained almost constant at $\simeq 50$ solar radius $\left(R_{\odot}\right)$ (Fig. 1-A). The stellar luminosity was substantially lower than the accretion luminosity (Fig. 1-B) and the KH timescale was much longer than the accretion time (Fig. 1. C). Consequently, entropy carried by the accreted gas accumulated at the stellar surface nearly without loss. During this quasi-adiabatic stage $\left(M_{*}<7 M_{\odot}\right)$, the luminosity $L_{*}$ increased with stellar mass. When the star grew to $8 M_{\odot}$, the KH timescale finally fell below the accretion timescale (Fig. 1.C). After this, the protostar began its so-called $\mathrm{KH}$ contraction, in which it gradually contracted as it radiated its energy away (Fig. 1-A). The stellar luminosity was the main component of the total luminosity after this evolutionary stage (Fig. 1.B). The stellar luminosity $L_{*}$ increased, and stellar radius $R_{*}$ decreased, as the stellar mass increased. As a result, the stellar effective temperature $T_{\text {eff }} \propto\left(L_{*} / R_{*}^{2}\right)^{1 / 4}$ and the ultraviolet (UV) flux rapidly rose (Fig. 1.D). Thus, ionization and heating by UV photons became important already in the KH contraction stage.

When the stellar mass was $20 M_{\odot}$, an ionized region rapidly expanded in a bipolar shape perpendicular 
to the disk, where gas was cleared away (Fig. 2-A). At this moment, the disk extended out to $\simeq 600$ AU. The disk was self-shielded against the stellar $\mathrm{H}_{2}$-dissociating $(11.2 \mathrm{eV} \leq h \nu \leq 13.6 \mathrm{eV})$ as well as the ionizing radiation. The ionized atomic hydrogen (HII) region continued to grow and finally broke out of the accreting envelope. At $M_{*} \simeq 25 M_{\odot}$, the size of the bipolar HII region exceeded $0.1 \mathrm{pc}$ (Fig. 2-B). Because of the high pressure of the heated ionized gas, the opening angle of the ionized region also increased as the star grew (Fig. 2-C). Shocks propagated into the envelope preceding the expansion of the ionized region. The shocked gas was accelerated outward at a velocity of several kilometers per second. The shock even reached regions shielded against direct stellar UV irradiation. The outflowing gas stopped the infall of material from the envelope onto the disk (Fig. S6). Without the replenishment of disk material from the envelope the accretion rate onto the protostar decreased (Fig. 31). In addition, the absence of accreting material onto the circumstellar disk means that the disk was exposed to the intense ionizing radiation from the star. The resulting photoevaporation of disk gas also reduced the accretion rate onto the protostar. The photoevaporated gas escaped toward the polar direction within the ionized region. The typical velocity of the flow was several tens of kilometers per second, comparable with the sound speed of the ionized gas, which was high enough for the evaporating flow to escape from the gravitational potential well of the dark matter halo.

When central nuclear hydrogen burning first commenced at a stellar mass of $35 M_{\odot}$, it was via the proton-proton (pp) -chain normally associated with low-mass stars. The primordial material does not have the nuclear catalysts necessary for carbon-nitrogen-oxygen (CNO) -cycle hydrogen burning. Because the pp-chain alone cannot produce nuclear energy at the rate necessary to cover the radiative energy loss from the stellar surface, the star continues to contract until central temperatures and densities attain values that enable the 3- $\alpha$ process of helium burning (11). The product of helium burning is carbon, and once the relative mass abundance of carbon reaches $\sim 10^{-12}$, CNO-cycle hydrogen burning takes over as the principal source of nuclear energy production, albeit at much higher central densities and temperatures than those of stars with solar abundances. These first-generation ZAMS (zero-age main sequence) stars are thus more compact and hotter than their present-day counterparts of equal mass (14). The subsequent evolution of the accreting star followed along the ZAMS mass-radius relationship (Figure [1A). By the time the star attained $40 M_{\odot}$, the entire region above and below the disk (Fig. 2D) was ionized. Mass accretion was terminated when the stellar mass was $43 M_{\odot}$ (Fig. 3).

The entire evolution described above took about 0.1 million years from the birth of the embryo protostar to the termination of the accretion. The star is expected to live another few million years before exhausting all available nuclear fuel and exploding as a core-collapse supernova (15).

Our calculations show that the first stars regulated their growth by their own radiation. They were not extremely massive, but rather similar in mass to the O-type stars in our Galaxy. This resolves a long-standing enigma regarding the elemental abundance patterns of the Galactic oldest metal-poor stars, which contain nucleosynthetic signatures from the earliest generation of stars. If a substantial number of first stars had masses in excess of $100 M_{\odot}$, they would end their lives through pair-instability supernovae $(16,17)$, expelling heavy elements that would imprint a characteristic nucleosynthetic signature to the elemental abundances in metal-poor stars. However, no such signatures have been detected in the metalpoor stars in the Galactic halo $(18,19)$. For example, the abundances of elements with odd atomic numbers are generally reduced in remnants of primordial supernovae. The odd-even contrast pattern expected in pair-instability supernovae is much stronger than the observed patterns in Galactic metalpoor stars $(17)$. Moreover, pair-instability supernovae predict a small abundance ratio $[\mathrm{Zn} / \mathrm{Fe}]$, but observed values are much larger (16). Detailed spectroscopic studies of extremely metal-deficient stars indicate that the metal-poor stars were born in an interstellar medium that had been metal-enriched by supernovae of ordinary massive stars (20).

Second-generation stars, which formed from the primordial gas affected by radiative or mechanical feedback from the first stars, could have dominated the metallicity of the young interstellar medium, which then spawned the observed Galactic halo stars. These second-generation stars could have been 
more numerous but less massive than the first stars because of a different gas thermal evolution, with additional radiative cooling via $\mathrm{H}_{2}$ and $\mathrm{HD}$ molecules (21, 22). However, this mode of star formation is suppressed even with weak $\mathrm{H}_{2}$ photodissociating background radiation (22). If so suppressed, the formation process of the later-generation primordial stars would be similar to that of the very first stars, and most primordial stars could have experienced the evolution presented in this article regardless of their generation. One might argue that today's observed metal-deficient stars formed after an episode of star formation with non-zero metallicity. For the star formation process to differ substantially from that of the very first stars, one would require metallicities in excess of $[\mathrm{Fe} / \mathrm{H}]>-5$ (23, 24). Caffau and co-workers (25) report on observations of a metal-deficient star with $[\mathrm{Fe} / \mathrm{H}] \simeq-5$ but without the corresponding enhancement of carbon, nitrogen, and oxygen found in metal-deficient stars. The abundance pattern of this star agrees with expectations from core-collapse supernovae, implying that it formed from gas enhanced by material ejected from primordial stars with masses less than $100 M_{\odot}$.

We have performed radiation-hydrodynamic simulations only for a single star-forming region embedded in a cosmological simulation. Our selected dark halo was typical in mass, spin, and formation epoch, when compared with those in other studies (5). The evolution presented here is somewhat similar to that predicted by the semi-analytic model, in which the expansion of the ionized region begins when the stellar mass is $\simeq 25 M_{\odot}$ and the final mass is $\simeq 57 M_{\odot}(8)$, the lowest final stellar mass predicted by the semi-analytic treatment. If input parameters of the semi-analytic models are chosen to fit our initial gas cloud, however, the final mass should be higher, $\simeq 90 M_{\odot}$ (SOM text). Our calculations follow the dynamical response of the infalling gas onto the circumstellar disk. The expansion of the ionized region around the protostar generates a powerful outflow even behind the surrounding disk. This effect reduces the accretion rate significantly. Thus, our radiation-hydrodynamic calculations predict systematically lower final masses than those of the semi-analytic models.

Although the results described above provide a complete picture of how a primordial protostar regulates and terminates its growth, there are a few key quantities that determine the strength of the feedback effect, as suggested by the semi-analytic model (8). With smaller initial rotation of the natal dense core, the density in the envelope near the polar directions would be higher. Hydrogen recombination occurs rapidly in the dense gas, which prevents the breakout of the ionized region. Gas accretion can last for a longer time in this case, and would form more massive stars (SOM text). Nevertheless, even considering variations among dark halos bearing the first stars, a substantial fraction of the first stars should be less massive than $100 M_{\odot}$ and end their lives as ordinary core-collapse supernovae. Gas accretion might not be completely halted in a few exceptional cases, thus leading to the formation of a small number of extremely massive stars that are $>100 M_{\odot}$ in the early universe (26). Black holes left after such very massive stars' deaths might have grown up to be the supermassive black holes lying in galaxies.

Recent 3D cosmological simulations showed that a primordial gas cloud breaks up into several embryo protostars in an early phase $(27,28)$. Each of these protostars would continue to grow through mass accretion, but it remained uncertain how and when the growth is halted. Our radiation-hydrodynamics calculations explicitly show that the parent gas cloud is evaporated by intense radiation from the central star when its mass is several tens of solar-masses. The circumstellar disks in our simulations are marginally stable against gravitational fragmentation [Fig. S5]. We expect that these disks - in a 3D simulation - would evolve analogously to our numerical simulations with assumed axial symmetry and have a similar time-averaged structure. It is conceivable that a few protostars are ejected dynamically from the parent cloud, to remain as low-mass stars. Observationally, however, there have been no low-mass zero-metallicity stars discovered in the Galaxy. This fact suggests the limited formation efficiency of such low-mass primordial stars $(19)$. Low-mass stars $\left(<1 M_{\odot}\right)$ and extremely high mass stars $\left(>100 M_{\odot}\right)$, if any, are thus a minor population among the first stars.

Our self-consistent calculations show that the characteristic mass of the first stars is several tens of solar masses. Although this is less than that of the conventionally proclaimed extremely massive stars $\left(>100 M_{\odot}\right)$, it is still much larger than the characteristic mass of stars in our galaxy $\left(<1 M_{\odot}\right)(29)$. 


\section{References and Notes}

1. V. Bromm, N. Yoshida, L. Hernquist, C. F. McKee, Nature 459, 49 (2009).

2. K. Omukai, R. Nishi, Astrophys. J. 508, 141 (1998).

3. T. Abel, G. L. Bryan, M. L. Norman, Science 295, 93 (2002).

4. N. Yoshida, K. Omukai, L. Hernquist, T. Abel, Astrophys. J. 652, 6 (2006).

5. B. W. O'Shea, M. L. Norman, Astrophys. J. 654, 66 (2007).

6. N. Yoshida, K. Omukai, L. Hernquist, Science 321, 669 (2008).

7. H. W. Yorke, P. Bodenheimer, Astrophys. J. 525, 330 (1999).

8. C. F. McKee, J. C. Tan, Astrophys. J. 681, 771 (2008).

9. H. W. Yorke, A. Welz, Astron. 83 Astrophys. 315, 555 (1996).

10. H. W. Yorke, C. Sonnhalter, Astrophys. J. 569, 846 (2002).

11. K. Omukai, F. Palla, Astrophys. J. 589, 677 (2003).

12. T. Hosokawa, K. Omukai, Astrophys. J. 691, 823 (2009).

13. This definition of accretion luminosity includes the mechanical luminosity of an accretion-driven wind and the ratio $t_{\mathrm{KH}} / t_{\mathrm{acc}}$ is equal to the ratio $L_{\mathrm{acc}} / L_{*}$.

14. D. Ezer, A. G. W. Cameron, Astrophys. ES Space Science 14, 399 (1971).

15. D. Schaerer, Astron. \& Astrophys. 382, 28 (2002).

16. H. Umeda, K. Nomoto, Astrophys. J. 565, 385 (2002).

17. A. Heger, S. E. Woosley, Astrophys. J. 567, 532 (2002).

18. J. Tumlinson, A. Venkatesan, J. M. Shull, Astrophys. J. 612, 602 (2004).

19. A. Frebel, J. L. Johnson, V. Bromm, Mon. Not. R. Astron. Soc. 392, L50 (2009).

20. N. Iwamoto, H. Umeda, N. Tominaga, K. Nomoto, K. Maeda, Science 309, 451 (2005).

21. B. W. O'Shea, T. Abel, D. Whalen, M. L. Norman, Astrophys. J. Lett. 628, L5 (2005).

22. N. Yoshida, K. Omukai, L. Hernquist, Astrophys. J. Lett. 667, L117 (2007).

23. $[\mathrm{Fe} / \mathrm{H}] \equiv \log (\mathrm{Fe} / \mathrm{H})_{\text {star }}-\log (\mathrm{Fe} / \mathrm{H})_{\text {sun }}$, where $(\mathrm{Fe} / \mathrm{H})$ is mass ratio of $\mathrm{Fe}$ to $\mathrm{H}$.

24. T. Hosokawa, K. Omukai, Astrophys. J. 703, 1810 (2009).

25. E. Caffau, et al., Nature 477, 67 (2011).

26. T. Ohkubo, K. Nomoto, H. Umeda, N. Yoshida, S. Tsuruta, Astrophys. J. 706, 1184 (2009).

27. M. J. Turk, T. Abel, B. O'Shea, Science 325, 601 (2009).

28. P. C. Clark, et al., Science 331, 1040 (2011).

29. G. Chabrier, Pub. Astron. Soc. Pac. 115, 763 (2003). 
30. S. Richling, H. W. Yorke, Astrophys. J. 539, 258 (2000).

31. H. W. Yorke, M. Kaisig, Computer Physics Communications 89, 29 (1995).

32. D. E. Osterbrock, G. J. Ferland, Astrophysics of gaseous nebulae and active galactic nuclei (2006).

33. D. Hollenbach, C. F. McKee, Astrophys. J. Suppl. Ser. 41, 555 (1979).

34. P. R. Shapiro, H. Kang, Astrophys. J. 318, 32 (1987).

35. P. Anninos, Y. Zhang, T. Abel, M. L. Norman, New Astronomy 2, 209 (1997).

36. C. D. Levermore, G. C. Pomraning, Astrophys. J. 248, 321 (1981).

37. N. J. Turner, J. M. Stone, Astrophys. J. Suppl. Ser. 135, 95 (2001).

38. M. Mayer, W. J. Duschl, Mon. Not. R. Astron. Soc. 358, 614 (2005).

39. B. T. Draine, F. Bertoldi, Astrophys. J. 468, 269 (1996).

40. J. C. Tan, C. F. McKee, Astrophys. J. 603, 383 (2004).

41. T. Abel, P. Anninos, Y. Zhang, M. L. Norman, New Astronomy 2, 181 (1997).

42. D. Galli, F. Palla, Astron. ES Astrophys. 335, 403 (1998).

43. F. Palla, E. E. Salpeter, S. W. Stahler, Astrophys. J. 271, 632 (1983).

44. A. G. G. M. Tielens, D. Hollenbach, Astrophys. J. 291, 722 (1985).

45. A. Stacy, T. H. Greif, V. Bromm, Mon. Not. R. Astron. Soc. 403, 45 (2010).

46. N. I. Shakura, R. A. Sunyaev, Astron. E Astrophys. 24, 337 (1973).

47. M. R. Krumholz, R. I. Klein, C. F. McKee, S. S. R. Offner, A. J. Cunningham, Science 323, 754 (2009).

48. T. Hosokawa, H. W. Yorke, K. Omukai, Astrophys. J. 721, 478 (2010).

49. S. W. Stahler, F. H. Shu, R. E. Taam, Astrophys. J. 241, 637 (1980).

50. M. N. Machida, K. Omukai, T. Matsumoto, S.-i. Inutsuka, Astrophys. J. Lett. 647, L1 (2006).

51. J. C. Tan, E. G. Blackman, Astrophys. J. 603, 401 (2004).

52. J. Silk, M. Langer, Mon. Not. R. Astron. Soc. 371, 444 (2006).

53. J. C. Tan, B. D. Smith, B. W. O'Shea, American Institute of Physics Conference Series, D. J. Whalen, V. Bromm, \& N. Yoshida, ed. (2010), vol. 1294 of American Institute of Physics Conference Series, pp. $34-40$.

54. M. N. Machida, S.-i. Inutsuka, T. Matsumoto, Astrophys. J. 724, 1006 (2010).

55. K. M. Kratter, C. D. Matzner, M. R. Krumholz, R. I. Klein, Astrophys. J. 708, 1585 (2010).

56. D. Hollenbach, D. Johnstone, S. Lizano, F. Shu, Astrophys. J. 428, 654 (1994).

57. F. D. Kahn, Astron. \& Astrophys. 37, 149 (1974).

58. H. W. Yorke, E. Kruegel, Astron. \&3 Astrophys. 54, 183 (1977). 
59. M. G. Wolfire, J. P. Cassinelli, Astrophys. J. 319, 850 (1987).

60. R. Kuiper, H. Klahr, H. Beuther, T. Henning, Astrophys. J. 722, 1556 (2010).

61. R. Kuiper, H. Klahr, H. Beuther, T. Henning, Astrophys. J. 732, 20 (2011).

62. T. Peters, et al., Astrophys. J. 711, 1017 (2010).

63. J. E. Dale, I. A. Bonnell, C. J. Clarke, M. R. Bate, Mon. Not. R. Astron. Soc. 358, 291 (2005).

64. T. Peters, R. S. Klessen, M.-M. Mac Low, R. Banerjee, Astrophys. J. 725, 134 (2010).

Acknowledgments: We thank T. Nakamura, K. Nomoto, S. Inutsuka, and N. Turner for stimulating discussions on this topic. Comments by an anonymous referee helped improve the manuscript. T.H. appreciates the support by Fellowship of the Japan Society for the Promotion of Science for Research Abroad. The present work is supported in part by the grants-in-aid by the Ministry of Education, Science and Culture of Japan (19047004, 2168407, 21244021:KO, 20674003:NY). Portions of this research were conducted at the Jet Propulsion Laboratory, California Institute of Technology, which is supported by NASA. Data analysis was (in part) carried out on the general-purpose PC farm at Center for Computational Astrophysics (CfCA) of National Astronomical Observatory of Japan.

\section{Supporting Online Material}

www.sciencemag.org

SOM text

Figures S1-S7

Tables S1 and S2

References (30-64)

25 April 2011; accepted 28 October 2011

Published online 10 November 2011 


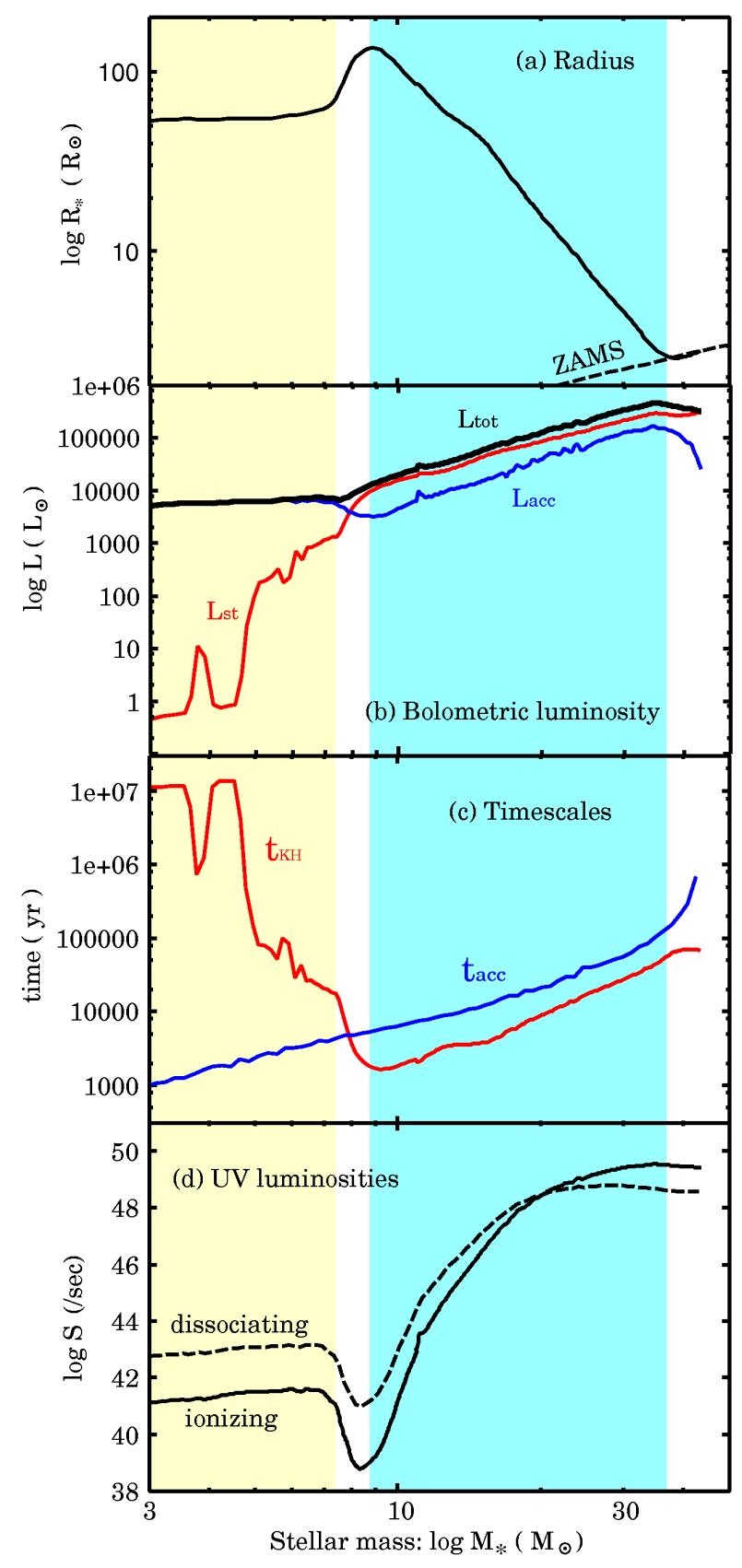

Fig. 1: Evolution of the stellar radius, bolometric luminosity, evolutionary timescales, and ionizing $(h \nu \geq 13.6 \mathrm{eV})$ and dissociating $(11 \mathrm{eV} \leq h \nu \leq 13.6 \mathrm{eV})$ photon number luminosity. In panel (b), the total luminosity $L_{\text {tot }}$ (black) is the sum of the stellar luminosity $L_{*}$ (red) and accretion luminosity $L_{\mathrm{acc}}$ (blue). The KH timescale $t_{\mathrm{KH}}$ (red) and accretion timescale $t_{\mathrm{acc}}$ (blue) are depicted in panel (c). The yellow and blue backgrounds denote the adiabatic accretion phase and KH contraction phase in the protostellar evolution. 

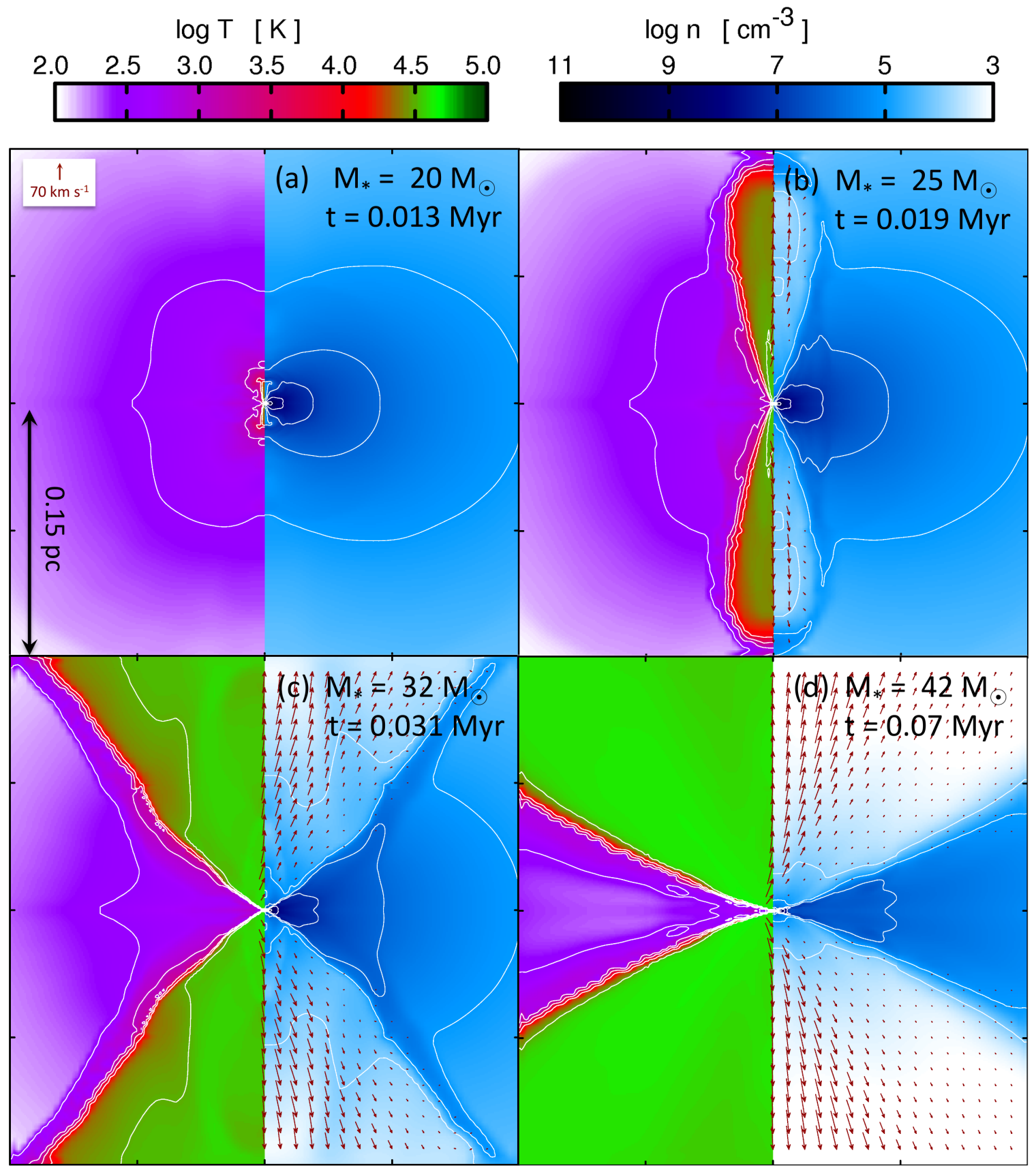

Fig. 2: UV radiative feedback from the primordial protostar. The spatial distributions of gas temperature (left), number density (right), and velocity (right, arrows) are presented in each panel for the central regions of the computational domain. The four panels show snapshots at times, when the stellar mass is $M_{*}=20 M_{\odot}(a), 25 M_{\odot}(b), 35 M_{\odot}(c)$, and $42 M_{\odot}(d)$. The elapsed time since the birth of the primordial protostar is labeled in each panel. 


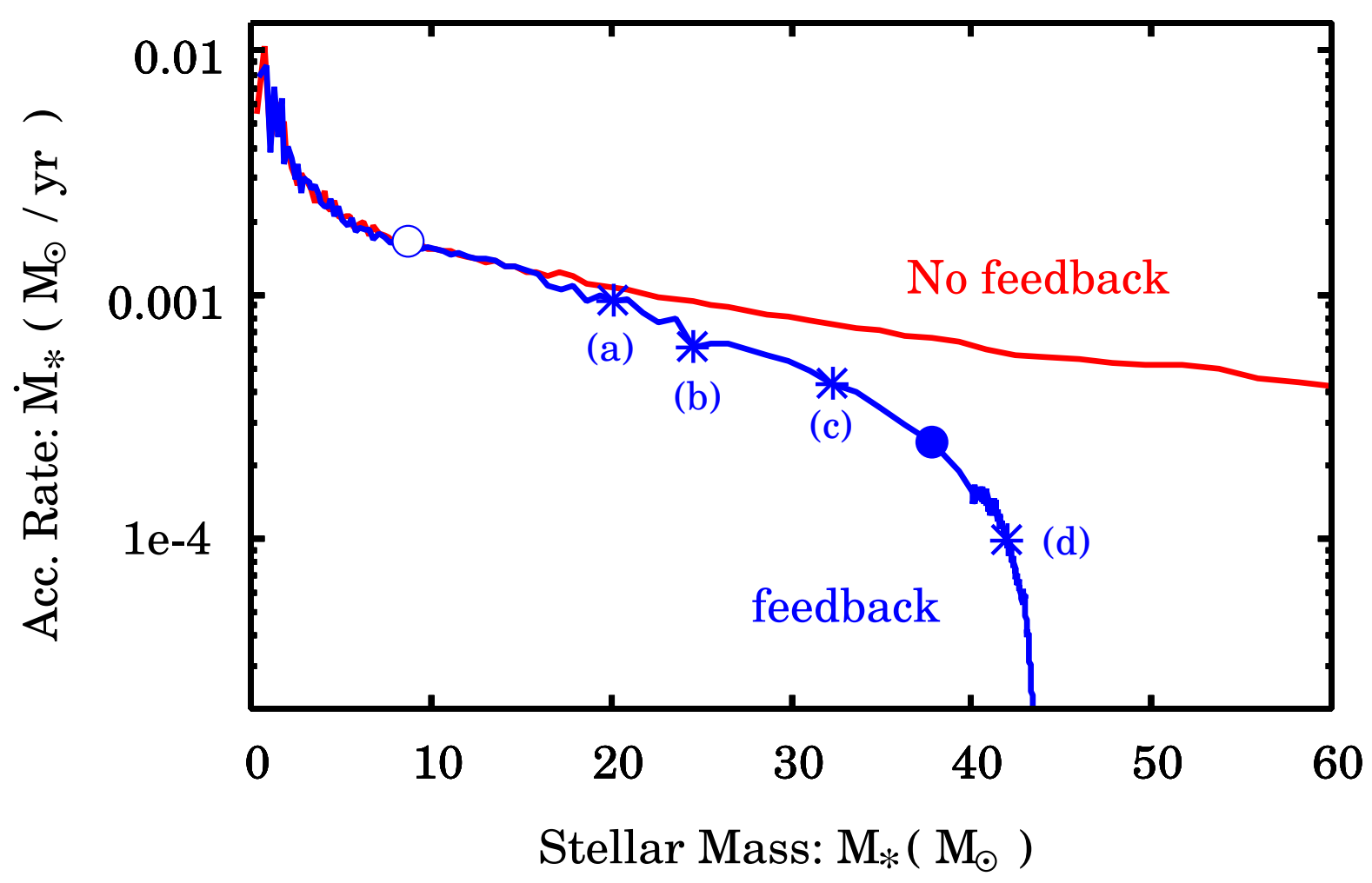

Fig. 3: Evolution of the accretion rate onto the primordial protostar. The blue line indicates the evolution, which includes the effect of UV radiative feedback from the protostar. The red line indicates a numerical experiment with no UV feedback. The open and solid circles denote the characteristic epochs of the protostellar evolution, beginning of the KH contraction and the protostar's arrival to the ZAMS. Figure 2. A to D, shows the snapshots at the moments marked here with asterisks. 


\section{Supporting Online Material}

\section{Numerical Methods}

We have developed a hybrid code to simultaneously calculate the evolution of an accreting first generation (proto-)star and the flow of the surrounding primordial material. We solve the accretion flow with a radiation hydrodynamic code, whereby the central (proto-)star is represented as a sink cell. This sink cell grows in mass by inflow through its boundary and it influences the surrounding material flow via gravity and by emitting radiation. We follow the evolution of the central star from the earliest protostellar stage up to the early phases of nuclear burning on the zero age main sequence by solving the conventional stellar structure equations, taking into account mass accretion. The material functions for the stellar evolution calculation - the opacity, the equation of state and other thermodynamic relationships, as well as the nuclear reaction network - assume zero metallicity. The calculated evolution provides the emitted radiation from the sink cell as a function of time. We describe below our methods of calculation for the accretion flow and the protostar, respectively.

\subsection{Radiation Hydrodynamics of the Accretion Flow}

To study the evolution of the flow of primordial gas we employ a grid-based axisymmetric radiation hydrodynamic code with self-gravity, previously used for studying present-day star formation $(7,10)$ and the evolution of photoionized gas flow from protostellar disks $(9,30)$. This code makes use of a nested-grid technique to cover a wide spatial range (31). We have added a chemical network besides changes based on the nature of the primordial material.

The governing equations for gas dynamics in cylindrical coordinate $(R, Z)$ are,

$$
\begin{gathered}
\frac{\partial \rho}{\partial t}+\nabla \cdot(\rho \boldsymbol{v})=0, \\
\frac{\partial(\rho \boldsymbol{v})}{\partial t}+\nabla \cdot(\rho \boldsymbol{v} \otimes \boldsymbol{v})=-\rho \nabla \Phi-\nabla p+\frac{A^{2}}{\rho R^{3}} \boldsymbol{n}_{R}+\boldsymbol{K} \\
\frac{\partial e}{\partial t}+\nabla \cdot(e \boldsymbol{v})=-p \nabla \cdot \boldsymbol{v}+\Gamma-\Lambda, \\
p=(\gamma-1) e,
\end{gathered}
$$

where $\rho$ and $p$ are the gas density and pressure, $\Phi$ the gravitational potential, $\boldsymbol{v}$ the 2-dimensional (2D) velocity vector $\left(v_{R}, v_{Z}\right), A \equiv \rho R v_{\phi}$ the angular momentum per unit volume, $\boldsymbol{n}_{R}$ the radial unit vector, $\boldsymbol{K}$ the radiation force, $e$ the gas internal energy density, $\Gamma$ and $\Lambda$ the heating and cooling rates per unit volume, and $\gamma$ the adiabatic exponent. We calculate $\gamma$ from the chemical composition at each grid cell as in (2). The radiative and chemical processes included in the energy source term $\Gamma-\Lambda$ are summarized in Table S1.

\subsubsection{Radiation Transfer}

For the problem at hand photons in different wavelength regimes play vastly different roles in the thermal and chemical processes. For example, whereas gas cools via molecular hydrogen line emission and infrared/optical continuum radiation, photons with energies $h \nu \geq 13.6 \mathrm{eV}$ (EUV) photoionize atomic hydrogen and photons with energies $11.2 \mathrm{eV} \leq h \nu \leq 13.6 \mathrm{eV}$ (FUV) photodissociate molecular hydrogen. To treat their transfer properly without time-consuming calculations, we adopt different methods for each of the radiation components. 
Table S1. Included thermal processes

\begin{tabular}{llc}
\hline \hline & Processes & References \\
\hline \multirow{4}{*}{ Heating } & Photoionization & $(32)$ \\
& Photodissociation & $(33)$ \\
& $\mathrm{H}_{2}$ formation & $(33),(34)$ \\
& & \\
\hline \multirow{5}{*}{ Cooling } & $\mathrm{H}_{2}$ collisional excitation & $\S 1.1 .1$ \\
& $\mathrm{H}^{-}$free-bound emission & $\S 1.1 .1$ \\
& $\mathrm{H}_{2}$ collisional dissociation & $(33),(34)$ \\
& $\mathrm{H}$ collisional ionization & $(32)$ \\
& $\mathrm{H}$ collisional excitation & $(35)$ \\
& Compton scattering & $(35)$ \\
& HeII collisional excitation & $(35)$ \\
& & \\
\hline
\end{tabular}

Molecular Hydrogen Line Cooling The primary cooling process in low-temperature $(<\sim 8000 \mathrm{~K})$ primordial gas is line emission via rotational and vibrational transitions of hydrogen molecules. We adopt the fitting formula by (33) for the optically-thin limit for $n<\sim 10^{9} \mathrm{~cm}^{-3}$. Dense gas with $n>\sim 10^{9} \mathrm{~cm}^{-3}$ is opaque to $\mathrm{H}_{2}$ line emission, and the cooling rate is reduced by photon trapping. In this case the cooling rate is calculated by adapting the method of (4) to the case of axial symmetry. We sum up cooling rates for all possible transitions among the rotational levels from $J=0$ to 20 and vibrational levels $v=0,1,2$. The cooling rate by a transition for the optically thick case is obtained by multiplying the value for optically thin cooling by the escape probability. The escape probability $\beta_{\text {esc }}$ is evaluated as

$$
\beta_{\mathrm{esc}}=\frac{\beta\left(\tau_{R}\right)+\beta\left(\tau_{Z}\right)}{2},
$$

where $\beta(\tau)$ is

$$
\beta(\tau)=\frac{1-\exp (\tau)}{\tau},
$$

and $\tau_{R}$ and $\tau_{Z}$ are the optical depths of this transition along $R$ and $Z$ directions, respectively. We calculate the optical depths using the local velocity gradients (so-called Sobolev approximation)

$$
\tau_{q}=\alpha \frac{c_{s}}{\left|\partial v_{q} / \partial q\right|},
$$

where $q=R$ or $Z, \alpha$ is the absorption coefficient and $c_{s}$ is the sound speed.

For calculating the level-population of $\mathrm{H}_{2}$ molecules, we omit the excitation by absorbing stellar photons, which could potentially reduce the line cooling rate. In general, $\mathrm{H}_{2}$ molecules could be excited to (i) the Lyman-Werner bands by absorbing FUV photons or (ii) the higher rotational and vibrational levels by absorbing infrared photons. In our case, the process (i) would be minor because the disk is shielded against the stellar FUV radiation (also see Sec. 3). The process (ii) is negligible in most parts of the disk even with the radiation from the protostar.

Continuum Cooling During the collapse phase before the formation of a protostar, continuum cooling via $\mathrm{H}_{2}$ collision-induced emission (CIE) is important in the dense $\left(n>10^{13} \mathrm{~cm}^{-3}\right)$ molecular gas (2). 
However, we only find such dense gas inside the sink cell; it does not otherwise appear in our hydrodynamical calculation. Instead, cooling via $\mathrm{H}^{-}$free-bound emission becomes important in the nearly vertical flows onto the circumstellar disk (also see Sec. 3 below).

The continuum cooling rate $\Lambda_{c}$ in the gray approximation is written as

$$
\Lambda_{c}=c \rho \kappa_{P}\left(4 \pi B\left(T_{g}\right)-E_{c}\right),
$$

where $c$ is the speed of light, and $\kappa_{P}$ is the Planck mean opacity per unit mass. The continuum radiation energy density $E_{c}$ satisfies the equation

$$
\frac{\partial E_{c}}{\partial t}=-\nabla \cdot \boldsymbol{F}_{c}+\Lambda_{c}+j_{*},
$$

where $\boldsymbol{F}_{c}$ is the energy flux, and $j_{*}$ is the stellar source term. Using the total luminosity of the protostar $L_{\text {tot }}$, the stellar source term is given by

$$
j_{*}=\frac{L_{\mathrm{tot}}}{2 \pi \Delta R^{2} \Delta Z}
$$

where $\Delta R$ and $\Delta Z$ are the size of the sink cell, which is equal to the cell size at the finest grid-level, $12 \mathrm{AU}$. The source term is zero except for the sink cell. We solve equations (9) with the flux-limited diffusion (FLD) approximation (36) using the operator splitting technique with $d e / d t=-\Lambda_{c}$ (37). The FLD method adopts a closure relation between $\boldsymbol{F}_{c}$ and $E_{c}$

$$
\boldsymbol{F}_{c}=-\frac{c \lambda}{\rho \kappa_{R}} \nabla E_{c}
$$

where $\kappa_{R}$ is the Rosseland mean opacity, and $\lambda$ is the flux-limiter defined as

$$
\begin{gathered}
\lambda=\frac{2+s}{6+3 s+s^{2}}, \\
s=\frac{\left|\nabla E_{c}\right|}{E_{c} \rho \kappa_{R}} .
\end{gathered}
$$

We use the opacities $\kappa_{P}$ and $\kappa_{R}$ for primordial gas calculated by (38) in tabulated forms. As mentioned above, the most important continuum cooling is that via $\mathrm{H}^{-}$free-bound emission. Neutral gas falling onto the disk is heated up to $T_{g} \simeq 5 \times 10^{3} \mathrm{~K}$ by compressional heating, until thermal balance is achieved with this cooling process. In our calculation the accreting envelope is always optically thin to the non-UV continuum radiation and non-UV stellar radiation escapes without significant heating in the envelope.

Stellar EUV and FUV Radiation EUV radiation from the star ionizes the material in its immediate vicinity. Within the HII region, there are two components of the EUV field: the direct stellar component and the diffuse component emitted via recombinations directly into the ground state (i.e. no "on-thespot" approximation). We solve the transfer of these two EUV components separately following (30). We adopt a frequency-averaged approximation for each component, taking into account the difference in their mean energies, $h \bar{\nu}_{*}$ (stellar) and $h \bar{\nu}_{d}$ (diffuse).

To calculate the direct EUV field we cast a number of radial rays from the central star to the outer edge of the simulation box, along which the transfer equation for the direct EUV photon number flux $\mathcal{F}_{*}$ is solved:

$$
\nabla_{r} \cdot \mathcal{F}_{*}=-n(1-x) \sigma_{*} \mathcal{F}_{*},
$$

where $x$ is the degree of ionization and $\sigma_{*}$ is the absorption cross section per particle. The cross section $\sigma_{*}$ is a function of mean energy of the direct EUV photons, i.e., the effective temperature of the star $T_{\text {eff }}(32)$. 
We employ the FLD approximation for the diffuse component, whose transfer equation is

$$
\frac{\partial \mathcal{N}_{d}}{\partial t}=-\nabla \cdot \mathcal{F}_{d}+\alpha_{1}\left(T_{g}\right) n^{2} x^{2}-n(1-x) \sigma_{d} c \mathcal{N}_{d},
$$

where $\mathcal{N}_{d}$ is the diffuse EUV photon number density, $\alpha_{1}$ is the recombination coefficient to the ground state, and $\sigma_{d}$ is the absorption cross section. Under the approximation that the mean energy of the diffuse component depends only on the local gas temperature $T_{g}$, the cross section $\sigma_{d}$ becomes a function of $T_{g}$ (32). In the FLD approximation,

$$
\mathcal{F}_{d}=-\frac{c \lambda}{n(1-x) \sigma_{d}} \nabla \mathcal{N}_{d}
$$

in analogy to equation (11). The photoionization heating and recombination cooling rates are calculated for each component of EUV radiation separately.

As for the photodissociating FUV radiation, we only consider the stellar direct component. We calculate the photodissociation rate by multiplying the value for the optically-thin case by the selfshielding factor (39)

$$
f_{\mathrm{sh}}\left(N_{\mathrm{H}_{2}}\right)= \begin{cases}1 & \left(N_{\mathrm{H}_{2}}<N_{1}\right) \\ \left(N_{\mathrm{H}_{2}} / N_{1}\right)^{-3 / 4} & \left(N_{1}<N_{\mathrm{H}_{2}}<N_{2}\right) \\ 0 & \left(N_{\mathrm{H}_{2}}>N_{2}\right),\end{cases}
$$

where $N_{\mathrm{H}_{2}}$ is the $\mathrm{H}_{2}$ column density from the protostar to the point under consideration, $N_{1}=10^{14} \mathrm{~cm}^{-2}$, and $N_{2}=10^{22} \mathrm{~cm}^{-2}$. Although the original functional form of $f_{\mathrm{sh}}\left(N_{\mathrm{H}_{2}}\right)$ by (39) is for the range $N_{1}<N_{\mathrm{H}_{2}}<N_{2}$, we adopt the cut-off at $N_{\mathrm{H}_{2}}>N_{2}$, where the photodissociation rate falls sharply according to (39). In our calculation, the column density largely exceeds $N_{2}$ along the radial rays incident on the circumstellar disk.

As shown in Sec. 3 below, the innermost part of the disk at $R<$ several $10 \mathrm{AU}$ is not spatially resolved in our calculations. We expect the innermost part of the disk to be optically thick and shield the material near the disk mid-plane behind it from the stellar radiation $(40)$. We adopt the following treatment for modeling this effect. During the calculation, we evaluate the disk scale height $H(R) \equiv c_{s} / \Omega$ at each radius $R$ on the equator. We compute $i_{R, d}$, the $R$-index of the cell at the outer edge of the unresolved part, for which $H(R)<\Delta Z$. The unresolved part is assumed to be opaque to radial rays incident on the $\left(i_{R, d}+1\right)$-th cell on the equator up to a height $\Delta Z$, but transparent for the other rays.

Radiation Force The total radiation force $\boldsymbol{K}$ is calculated as the sum of contributions by the continuum radiation, direct and diffuse EUV radiation:

$$
\boldsymbol{K}=\frac{\rho \kappa_{R}}{c} \boldsymbol{F}_{c}+\frac{n(1-x) \sigma_{*}}{c} h \bar{\nu}_{*} \mathcal{F}_{*}+\frac{n(1-x) \sigma_{d}}{c} h \bar{\nu}_{d} \mathcal{F}_{d}
$$

Here, we omit contributions from FUV photons. In reality, the gas accretion envelope is opaque against Lyman- $\alpha$ photons emitted from the stellar atmosphere and HII region. Radiation pressure via the Lyman$\alpha$ scattering is exerted on the accretion envelope. With mass accretion through the circumstellar disk, in particular, photons are preferentially transferred toward the polar direction, where the gas density rapidly decreases as the mass accretion proceeds ("flashlight effect": c.f. (7)). The semi-analytic modeling by (8) shows that the Lyman- $\alpha$ pressure could influence the dynamics of infalling material near the rotation axis. As also discussed in (8), however, the radiation pressure via Lyman- $\alpha$ scattering would be significantly reduced once gas in polar directions is blown away and photons escape from the cavity. In this paper, we focus on the UV radiative feedback effects to derive upper limits of the stellar final masses. 
Table S2. Included chemical reactions

\begin{tabular}{llc}
\hline \hline No. & Reactions & References \\
\hline $\mathrm{R} 1$ & $\mathrm{H}+\mathrm{e} \rightarrow \mathrm{H}^{+}+2 \mathrm{e}$ & $(41)$ \\
$\mathrm{R} 2$ & $\mathrm{H}^{+}+\mathrm{e} \rightarrow \mathrm{H}+\gamma$ & $(32)$ \\
$\mathrm{R} 3$ & $\mathrm{H}^{-}+\mathrm{H} \rightarrow \mathrm{H}_{2}+\mathrm{e}$ & $(42)$ \\
$\mathrm{R} 4$ & $\mathrm{H}_{2}+\mathrm{H}^{+} \rightarrow \mathrm{H}_{2}^{+}+\mathrm{H}$ & $(42)$ \\
R5 & $\mathrm{H}_{2}+\mathrm{e} \rightarrow 2 \mathrm{H}+\mathrm{e}$ & $(42)$ \\
R6 & $\mathrm{H}_{2}+\mathrm{H} \rightarrow 3 \mathrm{H}$ & $(34)$ \\
$\mathrm{R} 7$ & $3 \mathrm{H} \rightarrow \mathrm{H}_{2}+\mathrm{H}$ & $(43)$ \\
$\mathrm{R} 8$ & $2 \mathrm{H}+\mathrm{H}_{2} \rightarrow 2 \mathrm{H}_{2}$ & $(43)$ \\
R9 & $2 \mathrm{H} \mathrm{H}_{2} \rightarrow \mathrm{H}+\mathrm{H}_{2}$ & $(43)$ \\
$\mathrm{R} 10$ & $\mathrm{H}+\mathrm{e} \rightarrow \mathrm{H}^{-}+\gamma$ & $(42)$ \\
R11 & $2 \mathrm{H} \rightarrow \mathrm{H}^{+}+\mathrm{e}+\mathrm{H}$ & $(43)$ \\
R12 & $\mathrm{H}+\gamma \rightarrow \mathrm{H}^{+}+\mathrm{e}$ & $(32)$ \\
$\mathrm{R} 13$ & $\mathrm{H}_{2}+\gamma \rightarrow 2 \mathrm{H}$ & $(44)$ and (17) \\
R14 & $\mathrm{H}^{-}+\mathrm{e} \rightarrow \mathrm{H}+2 \mathrm{e}$ & $(41)$ \\
$\mathrm{R} 15$ & $\mathrm{H}^{-}+\mathrm{H}^{+} \rightarrow 2 \mathrm{H}$ & $(42)$ \\
& & \\
\hline
\end{tabular}

\subsubsection{Chemical Reactions in the Primordial Gas}

We consider the five species of primordial gas $\mathrm{H}, \mathrm{H}^{+}$, e, $\mathrm{H}_{2}$, and $\mathrm{H}^{-}$, based on the minimal model of (41) with some additional reactions. The included reactions are summarized in Table $\mathrm{S} 2$. Except $\mathrm{H}^{-}$, we solve kinetic equations with an implicit difference scheme. $\mathrm{H}^{-}$is assumed to be in chemical equilibrium. Photodissociation of $\mathrm{H}^{-}$is omitted in the adopted chemical network. In our calculations the gas density is high enough that the collisional destruction processes (R4, 14, and 15) control its abundance. We curtail helium chemistry by assuming that helium is singly ionized in an HII region and atomic elsewhere. We do not include deuterium reactions because HD cooling is relevant only in low-temperature $\left(T_{g}<200 \mathrm{~K}\right)$ and low-density $\left(n<10^{8} \mathrm{~cm}^{-3}\right)$ gas, which does not appear in our simulations.

\subsubsection{Angular Momentum Transport in the Accretion Disk}

In an exactly axisymmetric system, angular momentum must be conserved. In reality, however, a massive circumstellar disk can develop a non-axisymmetric spiral pattern, which exerts a torque on the matter in the disk and transfers angular momentum outward. Recent 3-dimensional (3D) numerical simulations demonstrated that this mechanism operates in fact in the circumstellar disks of the first stars $(28,45)$. We mimic this effect by adopting the angular momentum transport via the so-called $\alpha$-viscosity $(46)$. The equation of angular momentum transport is thus given by

$$
\frac{\partial A}{\partial t}+\nabla \cdot(A \boldsymbol{v})=-\frac{1}{R} \frac{\partial}{\partial R}\left(R^{3} \eta \frac{\partial \Omega}{\partial R}\right),
$$

where $\Omega$ is angular velocity, $\eta=2 \alpha \rho c_{s}^{2} /(3 \Omega)$, and $\alpha$ is a dimensionless free parameter.

We assume that the $\alpha$-parameter depends on the height from the equator,

$$
\alpha(R, Z)=\alpha_{0} \exp \left(-\frac{Z}{H(R)}\right),
$$


where $\alpha_{0}$ is a constant. The effective values of the $\alpha$-parameter in rapidly accreting circumstellar disks are $\alpha \simeq 0.1-1$ as estimated from 3D simulations of present-day massive star formation $(47)$ as well as the formation of the first stars (28). In the fiducial case explained in the main article, we adopted $\alpha_{0}=0.6$.

Figure S1] shows the evolution of accretion rates onto the protostar for different values of $\alpha_{0}$. Although the evolution is qualitatively similar in all cases, with higher $\alpha_{0}$ angular momentum is transferred more rapidly and therefore accretion rates onto the protostar become higher. We see that the final stellar mass increases with $\alpha_{0}$. With the low value of $\alpha_{0}=0.3$, the final stellar mass is about $35 M_{\odot}$. This is close to the low-mass limit of $30 M_{\odot}$ predicted by (3), who expected that this amount of gas would accrete onto the protostar in a few thousand years, which is much shorter than the timescale of the protostellar evolution. Even with $\alpha_{0}$ as large as unity, on the other hand, the final mass reaches at most $M_{*} \simeq 50 M_{\odot}$. Note, however, that this does not exclude the possibility of formation of higher-mass stars. The semianalytic models predict that more massive stars would form with weaker initial rotation of the natal core (8) (also see Sec. (4). Although rare, stars exceeding $100 M_{\odot}$ might still form in such circumstances.

Figure S1 also shows the evolution in such a test case, whereby the initial angular momentum is artificially reduced to $30 \%$ of the original value 1 The final stellar mass is $\simeq 85 M_{\odot}$ in this case. The increase of the final mass is understood as follows. First, the gas density remains high in the accretion envelope in the polar directions for the same stellar mass. The stellar EUV photons are consumed more effectively by photoionization of neutral hydrogen generated via rapid recombination, which delays growth of an HII region. Second, the protostellar evolution differs from the fiducial case, because of the higher accretion rates. Figure S2 shows that, with the weaker rotation, the Kelvin-Helmholtz contraction stage is shifted to higher stellar masses. Correspondingly, the stellar EUV luminosity increases rapidly at a stage when the protostar is more massive than for the fiducial case. Because of these effects, the stellar UV feedback becomes effective at higher stellar masses. We note that for all examined cases, the mass accretion ceases soon after the protostar's arrival to the zero age main sequence.

\subsection{Protostellar Evolution}

We follow the evolution of the central protostar by solving the four stellar structure equations taking account of mass accretion $(11,12,48)$ :

$$
\begin{gathered}
\left(\frac{\partial r}{\partial M}\right)_{t}=\frac{1}{4 \pi \rho r^{2}}, \\
\left(\frac{\partial P}{\partial M}\right)_{t}=-\frac{G M}{4 \pi r^{4}}, \\
\left(\frac{\partial L}{\partial M}\right)_{t}=\epsilon-T\left(\frac{\partial s}{\partial t}\right)_{M} \\
\left(\frac{\partial s}{\partial M}\right)_{t}=\frac{G M}{4 \pi r^{4}}\left(\frac{\partial s}{\partial p}\right)_{T}\left(\frac{L}{L_{s}}-1\right) C,
\end{gathered}
$$

where $M$ is the Lagrangian mass coordinate, $\epsilon$ is the energy production rate by nuclear fusion, $s$ is the specific entropy, and $L_{s}$ is the radiative luminosity with adiabatic temperature gradient. The coefficient, $C$ in equation (24) is unity if $L<L_{s}$ (i.e., in radiative layers), and given by the mixing-length theory if $L>L_{s}$ (i.e., in convective layers). We also solve the structure of the accretion flow inside the sink cell under the assumption of steady state and spherical symmetry. The entire structure of both the protostar and accretion flow is consistently determined to satisfy the jump conditions for the accretion shock at the stellar surface (49). Mass accretion rates onto the protostar $\dot{M}_{*}$ are given by mass inflow rates through

\footnotetext{
${ }^{1}$ The angular momentum is reduced at the beginning of the $2 \mathrm{D}$ calculation, e.g., at a point when the central density is $\simeq 10^{6} \mathrm{~cm}^{-3}$ in the run-away collapse stage (see Sec. 2).
} 
the surface of the sink cell in the radiation-hydrodynamics calculation. With a high accretion rate of $\dot{M}_{*}>10^{-4} M_{\odot} \mathrm{yr}^{-1}$, the accretion flow just above the stellar surface becomes opaque to the stellar radiation under the assumption of perfect spherical symmetry. The photosphere is located far from the stellar surface, its temperature is reduced to $\simeq 6000 \mathrm{~K}$ even for very massive stars of $M_{*} \simeq 100 M_{\odot}$ (11). With the realistic disk accretion, however, the stellar surface at high latitude is not totally embedded in such an opaque flow and the UV radiation can freely radiate. For this reason, we evaluate the protostellar EUV and FUV photon number luminosities using the fluxes at the stellar surface as

$$
\begin{gathered}
S_{\mathrm{EUV}}=4 \pi R_{*}^{2} \int_{13.6 \mathrm{eV}}^{\infty} \frac{\pi B\left(T_{\mathrm{eff}}\right)}{h \nu} d \nu, \\
S_{\mathrm{FUV}}=4 \pi R_{*}^{2} \int_{11.2 \mathrm{eV}}^{13.6 \mathrm{eV}} \frac{\pi B\left(T_{\mathrm{eff}}\right)}{h \nu} d \nu,
\end{gathered}
$$

where $R_{*}$ is the stellar radius, $B\left(T_{\text {eff }}\right)$ is the Planck function, and $T_{\text {eff }}$ is defined as

$$
T_{\mathrm{eff}}=\left(\frac{L_{*}+L_{\mathrm{acc}}}{4 \pi \sigma R_{*}^{2}}\right)^{1 / 4},
$$

where $L_{*}$ is the stellar luminosity, $L_{\text {acc }} \equiv G M_{*} \dot{M}_{*} / R_{*}$ is the accretion luminosity, and $\sigma$ is StefanBoltzmann constant.

The stellar UV luminosities given by equations (25) - (27) assume that all the gas reaching the stellar surface releases its gravitational energy at the stellar surface. In reality, some fraction of the gravitational energy would be radiated away from the circumstellar disk with a lower effective radiation temperature than that of the stellar surface. Because the accretion luminosity $L_{\text {acc }}$ is only a few $10 \%$ of the stellar luminosity $L_{*}$, when the protostellar feedback begins to influence the mass accretion (see the main article), we do not expect a significant change in the overall accretion process.

The stellar EUV photons are consumed mostly by photoionizing the recombined hydrogen atoms within the HII region. As the recombination rate is proportional to the square of density, this consumption is most significant in the vicinity of the protostar. In our calculations, however, the flow structure very near the protostar is masked by our assumption of a sink cell. We evaluate the EUV consumption rate within the sink cell as follows. First, we consider the spherical "evacuation zone", whose radius $R_{\mathrm{ev}}$ is smaller than the gravitational radius for the ionized gas,

$$
R_{g, \mathrm{HII}} \equiv \frac{G M_{*}}{c_{s, \mathrm{HII}}^{2}} \simeq 100 \mathrm{AU}\left(\frac{M_{*}}{10 M_{\odot}}\right)\left(\frac{T_{\mathrm{HII}}}{10^{4} \mathrm{~K}}\right)^{-1}
$$

and larger than the size of the sink cell, $\simeq 10 \mathrm{AU}$. In our calculations the temperature of ionized gas $T_{\mathrm{HII}}$ is $\simeq 3 \times 10^{4} \mathrm{~K}$ just after formation of the HII region and rises slightly as the stellar mass increases. We adopt $R_{\mathrm{ev}}=30 \mathrm{AU}$ as a fiducial value. The density distribution within the evacuation zone should be well approximated as the free-fall flow whose radial density structure follows $\rho(r) \propto r^{-3 / 2}$. The consumption rate of EUV photons within this zone is analytically written as,

$$
S_{\mathrm{EUV}, \mathrm{ev}}=\frac{\alpha \dot{M}_{\mathrm{ev}}^{2}}{8 \pi \mu^{2} G M_{*}} \ln \left(\frac{R_{\mathrm{ev}}}{R_{*}}\right),
$$

where $\alpha$ is the total recombination rate, $\mu=\left(1+4 y_{\mathrm{He}}\right) m_{p}$ with the helium abundance $y_{\mathrm{He}}$ and proton mass $m_{p}$, and $\dot{M}_{\mathrm{ev}}$ is the mass inflow rate into this zone. We evaluate $\dot{M}_{\mathrm{ev}}$ using the gas inflow rate along the $Z$-axis,

$$
\dot{M}_{\mathrm{ev}}=4 \pi \rho_{z, \mathrm{ev}} R_{\mathrm{ev}}^{2} \sqrt{\frac{2 G M_{*}}{R_{\mathrm{ev}}}}
$$


where $\rho_{z, \text { ev }}$ is the gas density at $(R, Z)=\left(0, R_{\text {ev }}\right)$. We suppose that the HII region is quenched inside the evacuation zone when the stellar EUV luminosity $S_{\mathrm{EUV}}$ is less than the consumption rate $S_{\mathrm{EUV} \text {,ev }}$. We do not solve the EUV radiative transfer for such cases. After $S_{\mathrm{EUV}}$ exceeds $S_{\mathrm{EUV}}$,ev , we remove the limit of $S_{\mathrm{EUV}, e v}$ and assume that the EUV luminosity from the sink is $S_{\mathrm{EUV}}$.

We only marginally resolve the evacuation zone with the current grid resolution. The estimated value of $S_{\mathrm{EUV}, \text { ev }}$ depends on the grid size without resolving $R_{g, \mathrm{HII}}$, because free-fall flow is valid only inside of $R_{g, \mathrm{HII}}$. For test calculations with a $2 \times$ coarser innermost grid around the protostar, formation of the HII region begins for a bit higher stellar mass (by a few 10\%). Moreover, the flow structure within the evacuation zone could be complex. For instance, the protostellar outflow might be launched from the innermost part of the disk (50) with the help of magnetic field generated by dynamo amplification (51,52). This outflow would help the breakout of the HII region by clearing out materials close to the star in the polar directions. However, the evolution should depend on the detailed density structure in the outflowlaunching region, which controls the EUV consumption rate.

\section{Simulation Setup}

As the initial condition of our calculation, we assume the structure of a dense core in the run-away collapse from the cosmological simulation by (6). The calculation by (6) followed the entire evolution from the cosmological initial condition to the birth of a primordial protostar under the standard $\Lambda \mathrm{CDM}$ cosmology. A small protostar of $M_{*} \simeq 0.01 M_{\odot}$ forms at $10^{20} \mathrm{~cm}^{-3}$ as a result of the run-away collapse of a dense primordial-gas core at the cosmological redshift $z=14$. Specifically, we take the central $0.3 \mathrm{pc}$ cube around the density peak when the maximum density is $10^{6} \mathrm{~cm}^{-3}$ as our initial condition. We reduce the $3 \mathrm{D}$ data to an axisymmetric $2 \mathrm{D}$ distribution by averaging over azimuthal angles. Our simulation box contains the total gas mass of $\simeq 300 M_{\odot}$.

The numbers of the grid cells are initially $N_{Z} \times N_{R}=42 \times 42$, including 2 ghost cells in each direction. After we start our axisymmetric calculation, the dense core experiences continued gravitational collapse. We increase the grid resolution for the central collapsing region by successively adding finer nested grids as needed. With this nested-grid technique, we always resolve the minimum Jeans length by tens of grid cells. The increase of the grid-level is limited up to 8 owing to computational cost for following the subsequent accretion phase until the final stellar mass is fixed. We terminate the calculation of the run-away collapse when the minimum Jeans length becomes too short to be resolved with the finest grids. At this point we create a sink cell at the origin and calculate the subsequent accretion phase as described in Sec. 1. The central density at this moment is $\simeq 10^{12} \mathrm{~cm}^{-3}$, and the cell size at the finest grid-level is $\simeq 12 \mathrm{AU}$.

This recipe enables us to smoothly connect the $3 \mathrm{D}$ cosmological simulation using the particle method to a $2 \mathrm{D}$ (axial symmetry assumed) local simulation using the nested-grid method. We have confirmed that the evolution in our $2 \mathrm{D}$ calculation is reasonably consistent with the $3 \mathrm{D}$ results after the maximum density exceeds $10^{6} \mathrm{~cm}^{-3}$. For example, the upper panel of Figure S3 shows a comparison of the angularmomentum profiles for the $3 \mathrm{D}$ and $2 \mathrm{D}$ calculations using a parameter,

$$
f_{\mathrm{Kep}}\left(M_{r}\right) \equiv \frac{V_{\phi, r}}{V_{\mathrm{Kep}, \mathrm{r}}}, \quad V_{\phi, r}=\frac{l_{r}}{r}, V_{\mathrm{Kep}, r}=\sqrt{\frac{G M_{r}}{r}},
$$

where $M_{r}$ is the enclosed mass within radius $r$, and $l_{r}$ is specific angular momentum averaged over the spherical shell whose radius is $r$. The profile for the $2 \mathrm{D}$ simulation is within $20 \%$ offset from that in the $3 \mathrm{D}$ case taken from (6).

Although most of our simulations were done with the settings described above, we also calculated early evolution, turning off the stellar feedback, with the higher maximum grid-level of 9 . The spatial resolution for $R<240 \mathrm{AU}$ is doubled in this case and size of the finest cell is $\simeq 6 \mathrm{AU}$. Figure $\$ 1$ shows the 
evolution of the accretion rate until the stellar mass reaches $30 M_{\odot}$. The accretion rates are initially a bit lower with the higher central resolution but converge to our fiducial case as the stellar mass increases.

\section{Structure of the Circumstellar Disk}

Here, we examine the detailed structure of the circumstellar disk observed in our fiducial case. Figure S4 shows the 2D structure of the region within $1000 \mathrm{AU}$ of the protostar, when the stellar mass is $\simeq 10 M_{\odot}$ and $20 M_{\odot}$. The snapshot for the $10 M_{\odot}$ star shows the structure before the formation of an HII region. We see that the accreting gas hits the disk surface and heats up to $\simeq 4000 \mathrm{~K}$ by compression. Radiative cooling via $\mathrm{H}^{-}$free-bound emission operates here as explained in Sec. 1.1.1, Figure S5 displays the radial structure of the disk at this moment. The hydrogen is primarily in molecular form within the disk due to rapid formation via the gas phase three-body reaction. Note that the existence of $\mathrm{H}_{2}$ molecules and their contribution for radiative cooling are not included in the semi-analytic models. The total mass of this molecular gas is $\simeq 10 M_{\odot}$, which is comparable to the stellar mass. Gas on the equator at $R<1000 \mathrm{AU}$ has the rotational velocity more than $80 \%$ of the local Kepler value. The disk scale height is resolved on average except for the innermost part at $R<100$ AU. The profile of the Toomre Q-parameter, a measure of the gravitational stability of the disk, shows that the minimum value $Q_{\min }$ is almost unity. Thus, the disk is marginally gravitationally stable against the fragmentation. However, high-resolution $3 \mathrm{D}$ simulations demonstrate that the disk fragments due to gravitational instability in very early stage of the accretion phase (28). Note that "ring-like" fragmentation can be captured even in axisymmetric $2 \mathrm{D}$ simulations. The absence of fragmentation in our simulation is probably due to the limited spatial resolution and the large values of the viscous $\alpha$-parameter, $\alpha_{0} \geq 0.3$ (also see Sec. 1.1.3). In our case, the disk is also marginally stable in an earlier stage when the stellar mass is $\simeq 3 M_{\odot}$ (Fig. (S5). This is almost the same even with the doubled spatial resolution for $R<240$ AU. Even disks which experience fragmentation have a quasi-static structure with $Q_{\min } \simeq 1$, if averaged over many rotation periods. Such structure is well mimicked in our calculations.

The right panel in Figure S4 shows the 2D disk structure just after the birth of the HII region. We see a strong photoevaporating flow in the polar directions within the HII region. The stellar FUV luminosity has also increased significantly, but the $\mathrm{H}_{2}$ molecular disk still exists, shadowing the stellar photodissociating photons from the dense equatorial regions, evident in the disk's temperature and density distributions. The mass of the molecular gas is $\simeq 10 M_{\odot}$ at this moment.

\section{Comparison to Semi-analytic Models}

Our calculations show that mass accretion toward forming first stars is shut off via dynamical expansion of the HII region and photoevaporation of the circumstellar disk. This is qualitatively consistent with the picture predicted by the semi-analytic models (8). Their models show that the final stellar mass is $\simeq 145 M_{\odot}$ for their fiducial case; this value varies with different sets of input parameters. One of the input parameters is $f_{\text {Kep }}$ given by equation (31); their adopted fiducial value is 0.5 . Figure S3 shows that $f_{\text {Kep }} \simeq 0.6-0.7$ for our fiducial case and is lower than 0.5 for the weak-rotation case. For the semi-analytic model with $f_{\text {Kep }}>0.25$, however, the final stellar mass does not depend strongly on $f_{\text {Kep }}$, but rather on the entropy of the accreting gas, measured with a non-dimensional parameter

$$
K^{\prime} \equiv \frac{P / \rho^{\gamma}}{1.88 \times 10^{12} \operatorname{cgs}}=\left(\frac{T}{300 \mathrm{~K}}\right)\left(\frac{10^{4} \mathrm{~cm}^{-3}}{n}\right)^{0.1},
$$

where $\gamma=1.1$ is adopted for a typical value for the primordial gas (2).

The semi-analytic models use $K^{\prime}=1$ for their fiducial case and show that the final mass roughly scales as $K^{\prime 1.3}$. From Figure $\$ 3$ we see that $K^{\prime} \simeq 0.7$ for our fiducial case and $K^{\prime} \simeq 0.8-1$ for the weak-rotation 
case. The semi-analytic model with $K^{\prime}=0.7$ predicts a final mass $\simeq 90 M_{\odot}$, twice our value of $45 M_{\odot}$. The final mass $\simeq 85 M_{\odot}$ for our weak-rotation case is somewhat lower than the semi-analytic prediction $>108 M_{\odot}$ for $K^{\prime}>0.8$.

The initial conditions in our examined cases correspond to typical values for gas clouds bearing the first stars. Figure S1 shows that the accretion rates for the cases considered lie in the range $10^{-2}$ to $10^{-3} M_{\odot} \mathrm{yr}^{-1}$ for the $10 M_{\odot}$ star, comparable to that expected for 12 mini-halos using the semi-analytic models (53). However, our calculations attain systematically lower final masses than predicted by semianalytic models.

We examine possible reasons for this difference below. First, in our calculations, the mass ratio between the star and disk $f_{d} \equiv M_{\text {disk }} / M_{*}$ differs from the fiducial value in the semi-analytic models, $f_{d}=0.3$. As discussed in Sec. 3, for our fiducial case the mass of the molecular disk is $\simeq 10 M_{\odot}$, when the stellar mass is $10 M_{\odot}$, and would be even higher, if we include the outer atomic part. In 2D simulations, however, the value of $f_{d}$ depends on the degree of $\alpha$-viscosity, $\alpha_{0}$ (see Sec. 1.1.3). With the larger value $\alpha_{0}=1$, for example, the mass of the molecular disk for the $10 M_{\odot}$ star is reduced to $8 M_{\odot}$. To derive the appropriate value of $f_{d}$, we need detailed 3D numerical simulations which solve the transport of angular momentum in self-gravitating disks. Recent work on the Galactic star formation demonstrates that massive disks with $f_{d} \geq 1$ can form in some situations, though the value of $f_{d}$ varies with different initial conditions of gas cores $(54,55)$.

We contend, however, that differences in the stellar feedback process are principally responsible for the differences in final stellar mass. The semi-analytic models adopt the following formula for estimating the photoevaporation rate of the disk,

$$
\dot{M}_{\text {evp }}=4.1 \times 10^{-5}\left(\frac{S_{\mathrm{EUV}}}{10^{49} \mathrm{sec}^{-1}}\right)^{1 / 2}\left(\frac{T_{\mathrm{HII}}}{10^{4} \mathrm{~K}}\right)^{2 / 5}\left(\frac{M_{* \mathrm{~d}}}{100 M_{\odot}}\right)^{1 / 2} M_{\odot} \mathrm{yr}^{-1},
$$

where $M_{* d}=M_{*}+M_{\text {disk }}(56)$. For our fiducial case the photoevaporation rate is about $2 \sim 3 \times$ $10^{-4} M_{\odot} \mathrm{yr}^{-1}$, after the stellar mass exceeds $30 M_{\odot}$. By contrast, equation (33) estimates an evaporation rate of several $10^{-5} M_{\odot} \mathrm{yr}^{-1}$, using $T_{\mathrm{HII}}=5 \times 10^{4} \mathrm{~K}$ and the values of $S_{\mathrm{EUV}}$ and $M_{* \mathrm{~d}}$ from the simulation ( $M_{\text {disk }}$ is the mass of the molecular disk). We attribute this difference to the basic assumption of an infinitely thin disk for deriving equation (33) (56). As a result, only the diffuse EUV radiation (see Sec. 1.1.1) is accounted for in equation (33). In our calculations the stellar direct EUV radiation field impinging on the flared disk is primarily responsible for determining the photoevaporation rate. Even if the transfer of diffuse EUV radiation is turned off in the simulation, we do not see any significant change in the evolution.

Moreover, the semi-analytic models assume that, after the HII region forms, mass accretion onto the circumstellar disk still continues from regions shaded by the disk, where the stellar UV radiation is blocked. In this picture the stellar final mass is determined when the photoevaporation rate exceeds the mass accretion rate from the shaded regions. This does not agree with our simulations. It is true that the stellar UV radiation is blocked behind the disk as shown in Figure 2 in the main article. In our calculations, however, a shock front propagates into the accretion envelope shaded by the disk, as the HII region dynamically expands. Figure $\$$ 6 shows that there is the outward pressure gradient within the HII region due to the photoevaporating flow. As the HII region dynamically expands, the shocked accretion envelope behind the disk also obtains the same pressure gradient. As a result, the shocked gas is accelerated outward due to this pressure gradient at a velocity of several $\mathrm{km} / \mathrm{sec}$. This is shown in Figure [S7, We see that the gas at $R>$ a few $10^{3}$ AU is moving outward when the stellar mass exceeds $20 M_{\odot}$. The outflow rate of atomic gas via this horizontal motion is $9 \times 10^{-4} M_{\odot} \mathrm{yr}^{-1}$, when the stellar mass is $35 M_{\odot}$. The mass supply from the envelope to the disk is cut off by this moment 2 The isolated

\footnotetext{
${ }^{2}$ In our simulations, we adopt a semi-permeable outer boundary condition, which allows outflow but prohibits mass inflow through the boundary. In principle this boundary condition can ultimately limit mass accretion onto the disk. However, mass infall toward the disk is reversed and the gas flows outward through the outer boundary long before this limit is
} 
disk without replenishment from the envelope continues to lose gas by the photoevaporation. Ultimately, mass accretion onto the protostar ceases and the stellar final mass is fixed.

\section{$5 \quad$ Potential Effects of 3-Dimensionality}

For this study we have adopted an axisymmetric 2D code for calculating the dynamical evolution of the accreting flow. Recent 3D simulations for the present-day high-mass star formation are helpful for speculating on potential 3D effects.

A primary radiative feedback effect from Galactic high-mass stars is radiation pressure exerted on dust grains coupled with the gas accretion flow. Historically, this effect was first studied under spherical symmetry (57-59) and it was found that the accretion flow toward high-mass stars is prevented by the strong repulsive force (called as "radiation pressure problem"). 2D numerical simulations show that non-spherical mass accretion via disks significantly alleviates this problem (10,60). 3D simulations by $(47)$ demonstrate that 3D effects (e.g., radiative Rayleigh-Taylor instability growing in the accretion envelope) also reduce the repulsive force in addition to the effect of the disk accretion. However, it is still controversial whether the 3D effects are essential for determining the upper mass limit for present-day high-mass star formation. More recent 3D simulations by (61) show that the radiation pressure barrier is circumvented with the disk accretion, but find no critical 3D effects.

Without dust grains the opacity of the primordial gas is much lower than for the present-day Galactic interstellar medium. The dynamical evolution discussed in our article is not primarily due to radiation pressure, but rather to the high gas pressure of photoionized gas, which causes the dynamical expansion of the HII region supplemented by the photoevaporation of the disk. UV feedback effects in the present-day high-mass star forming regions have also been studied with 3D numerical simulations (62, 63). However, these studies focus on the feedback effects over the large length-scale of cluster-forming clumps, $\sim 1 \mathrm{pc}$. By contrast, we consider here the smaller-scale feedback processes in the vicinity of protostars, such as photoevaporation of the disks. The photoevaporation rate from the disk is expected to be highest at a

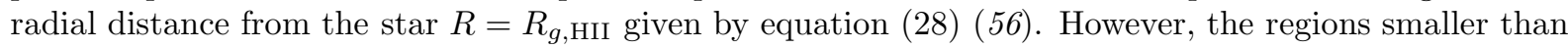
$1000 \mathrm{AU}$ around forming stars are not spatially resolved in the above-cited studies. The photoevaporation of the disk is a local process which takes place in the very central part of the HII region. In 3D we can expect the photoionized gas to have a clumpier structure than modeled by our $2 \mathrm{D}$ simulations. However, such small-scale inhomogeneities would be smoothed out within the sound crossing time, which is much shorter than the dynamical timescale of the expanding HII region.

As referred in Sec. 1.1.3, recent 3D simulations demonstrate that the circumstellar disks around primordial protostars fragment in the early stage of the accretion phase. The fragmentation of the disk would somewhat reduce the stellar final masses, as the accreting materials are shared among the fragments (64). Several authors have also suggested that a turbulent velocity field is present after the gravitational collapse of primordial gas cores (3). Although the expected turbulent field is not strong for the very first stars, we note that, in our case, the turbulent field is further weaken by mapping the $3 \mathrm{D}$ data to the $2 \mathrm{D}$ axisymmetric data (see Sec. 2). With a random velocity field the angular momentum directions of accreting materials would be time-dependent. As a result, the polar direction of the circumstellar disk should vary with time as well and the photoevaporating flow would clear out a larger amount of accreting materials than otherwise. We speculate that this effect would accelerate the horizontal expansion of the HII region and reduce the resulting final stellar mass. Our obtained final mass of the first stars, several $10 M_{\odot}$, is thus a conservative upper limit. Nonetheless, this is still much lower than the postulated high values exceeding $100 M_{\odot}$.

reached. 


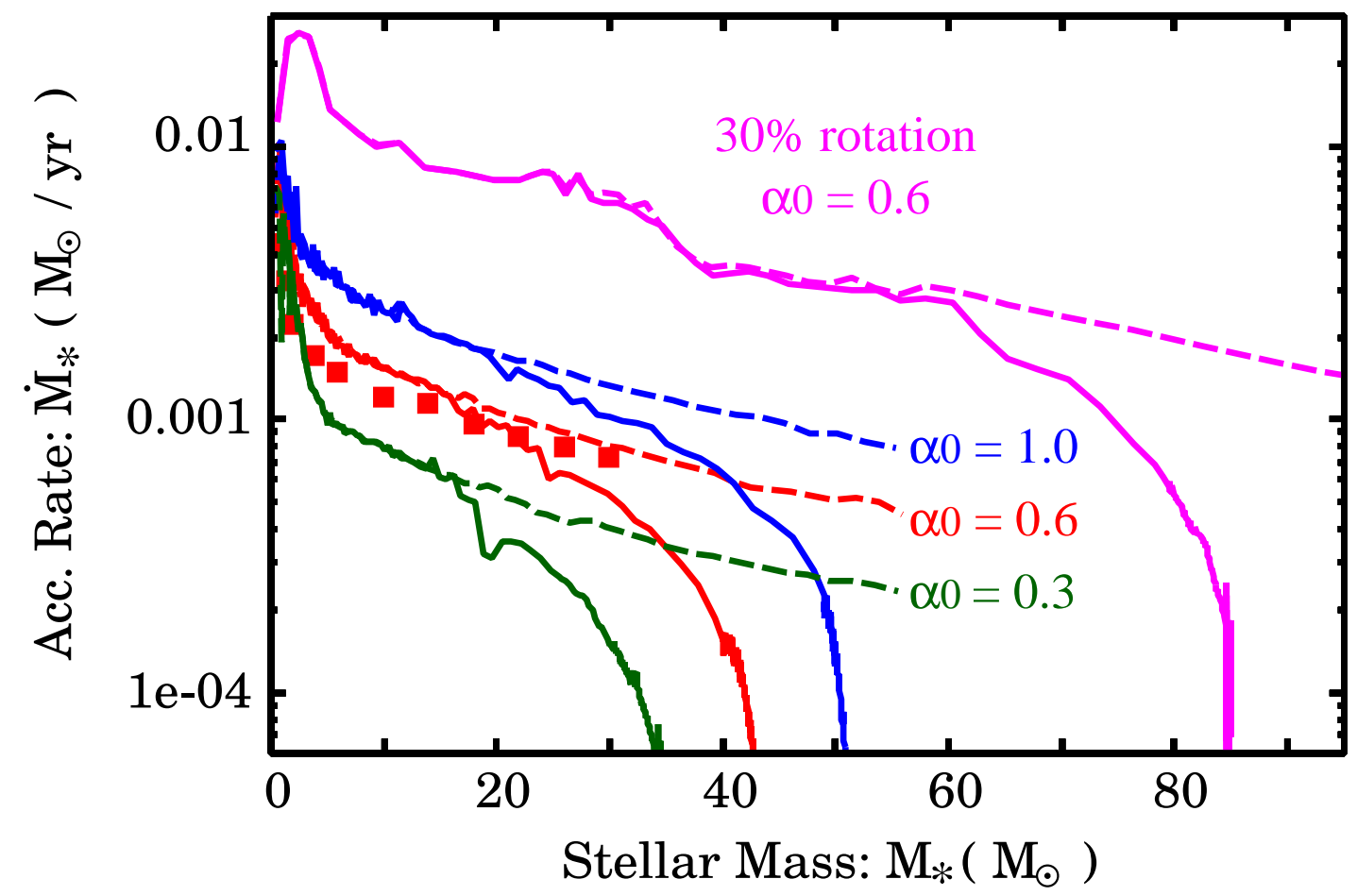

Fig. S1: Evolution of accretion rates onto the protostar with different $\alpha$-parameters for angular momentum transport. The blue, red, and green curves depict the results with $\alpha_{0}=1,0.6$, and 0.3 in equation (20), respectively. The magenta curves display the evolution with the initial angular momentum reduced to $30 \%$ of the fiducial value. For each case the solid and dashed lines represent the evolution with and without radiative feedback from the protostar, respectively. The red filled squares represent the nofeedback case with $\alpha_{0}=0.6$ doubling the spatial resolution in the central region of $R<240 \mathrm{AU}$ (also see Sec. 2). 


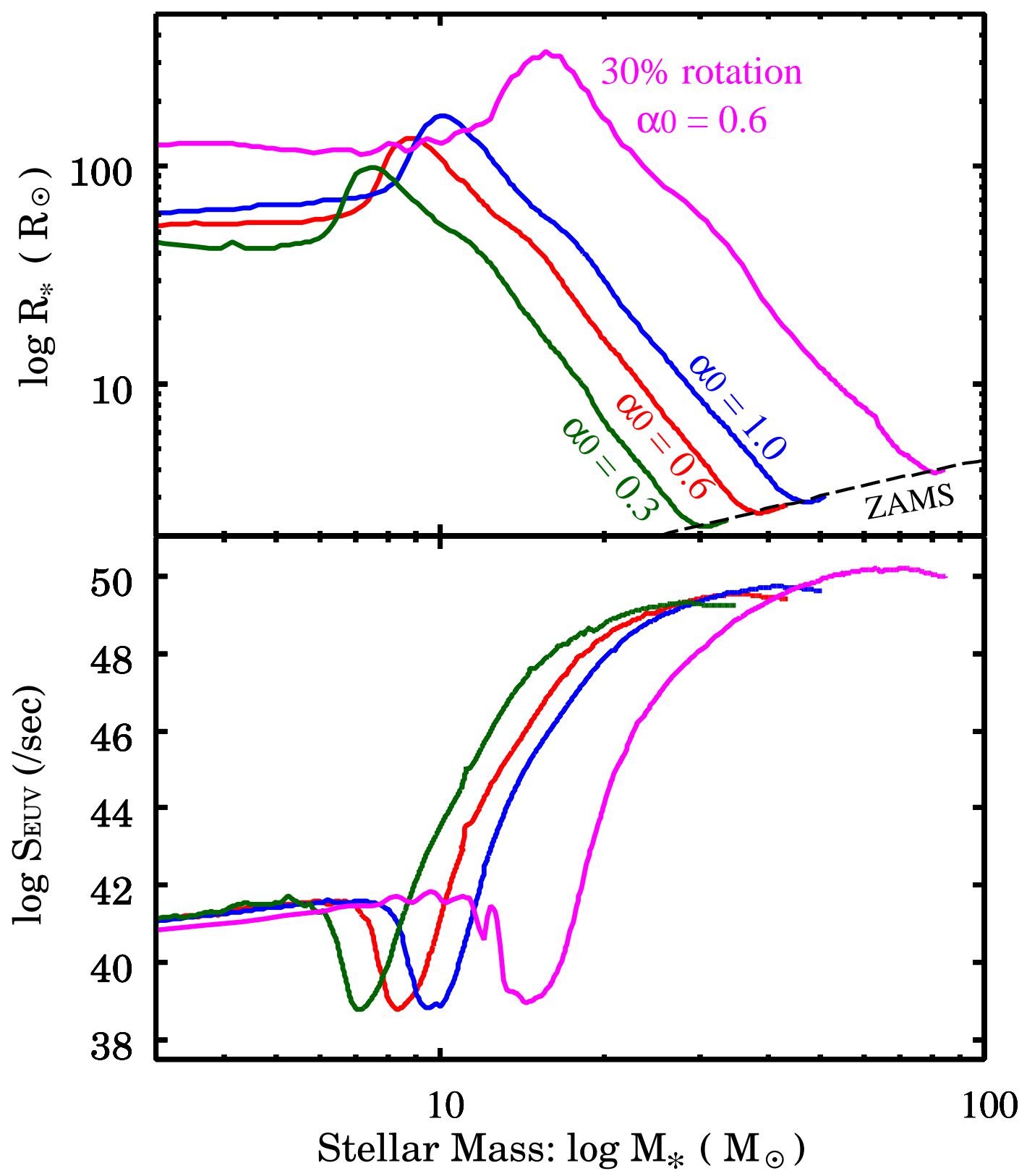

Fig. S2: Accretion rates onto the protostar as a function of the accreted stellar mass, assuming different $\alpha$-parameters for angular momentum transport. The blue, red, and green curves depict the evolution with $\alpha_{0}=1,0.6$, and 0.3 in equation (20), respectively. The magenta curves display the evolution with the initial angular momentum reduced to $30 \%$ of the fiducial value. For each case the solid and dashed lines represent the evolution with and without radiative feedback from the protostar, respectively. 


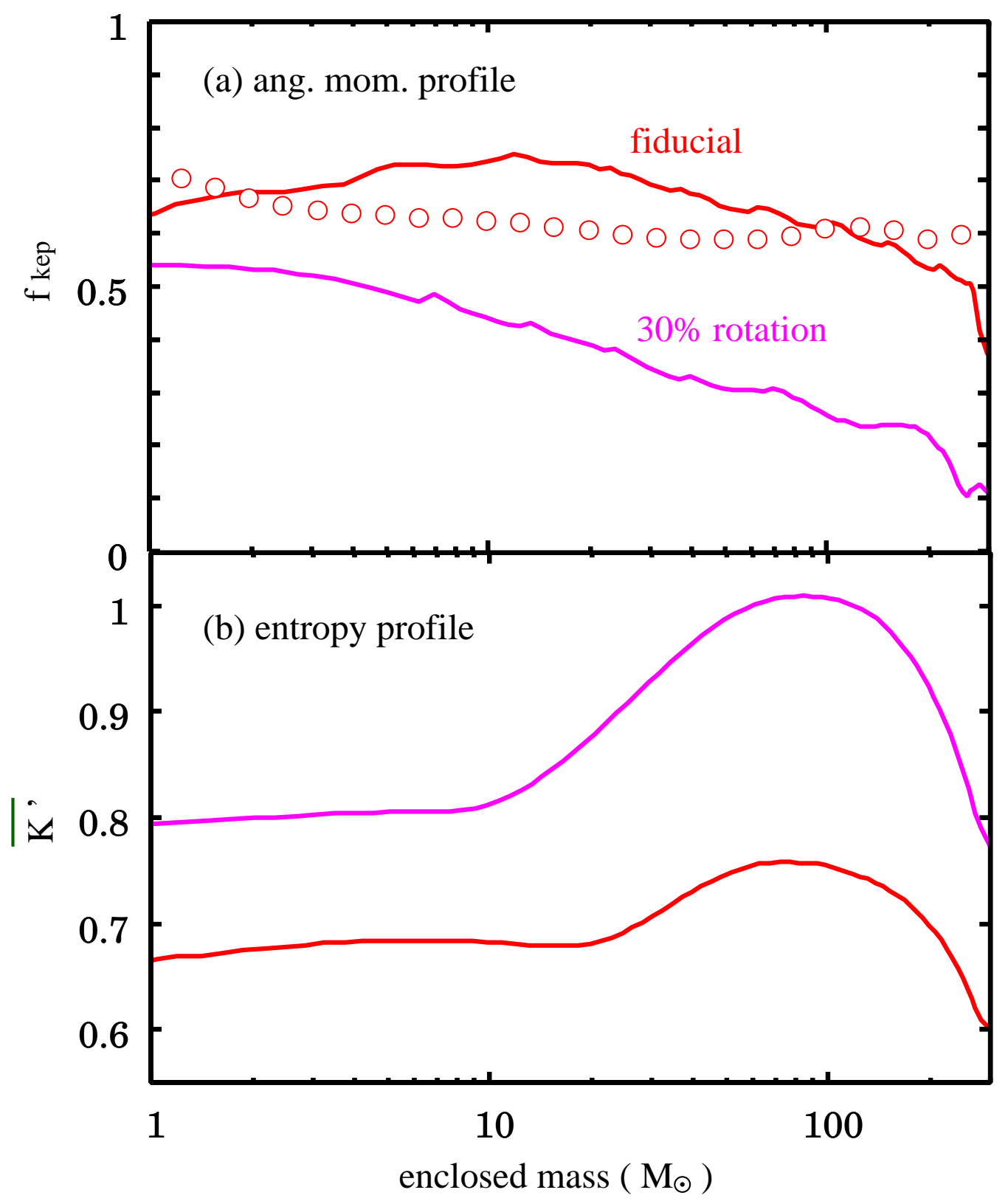

Fig. S3: Structure of the accreting envelope at the end of the calculation of the run-away collapse stage for the fiducial case (red lines) and weak-rotation case (magenta lines). Upper panel: the ratio of the local rotational velocity to Kepler velocity $f_{\text {kep }}$. The red open circles represent a snapshot at the birth of a embryo protostar in a three-dimensional cosmological simulation (6). Lower panel: Dimensionless entropy of the accreting gas $K^{\prime}$. The mass-weighted average values $\overline{K^{\prime}}$ are plotted against the enclosed gas mass. 


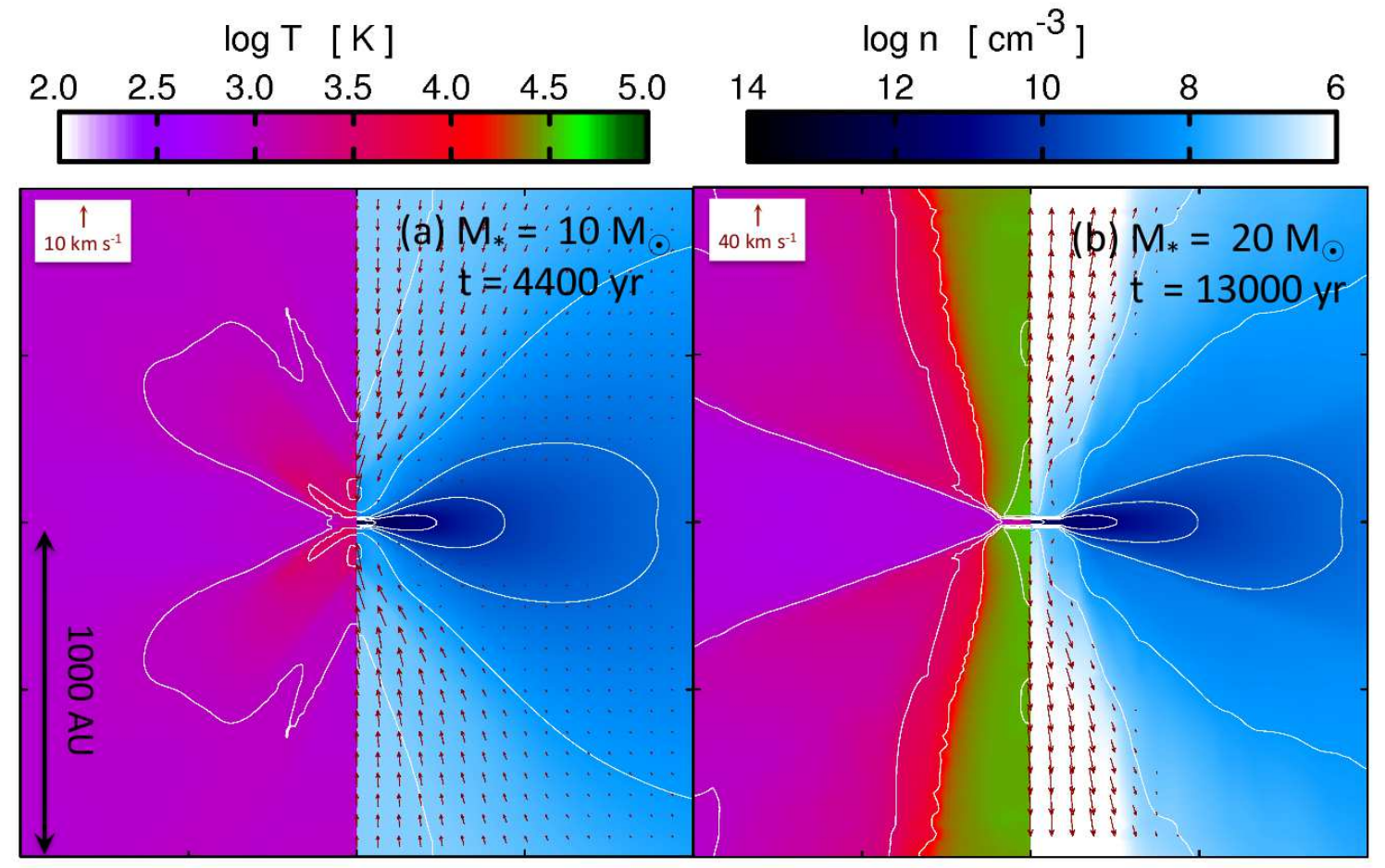

Fig. S4: The structure of gas temperature (left-hand side) and of number density and velocity (righthand side) in the vicinity of $1000 \mathrm{AU}$ around the protostar. The left and right panels show the snapshots when the stellar mass is $\simeq 10 M_{\odot}$ and $20 M_{\odot}$. The elapsed time since the birth of the protostar is also shown in each panel. Note that the color scale of the density, the legend of the velocity vectors, and size of the plotted area are different from those in Figure 2 in the main article. 


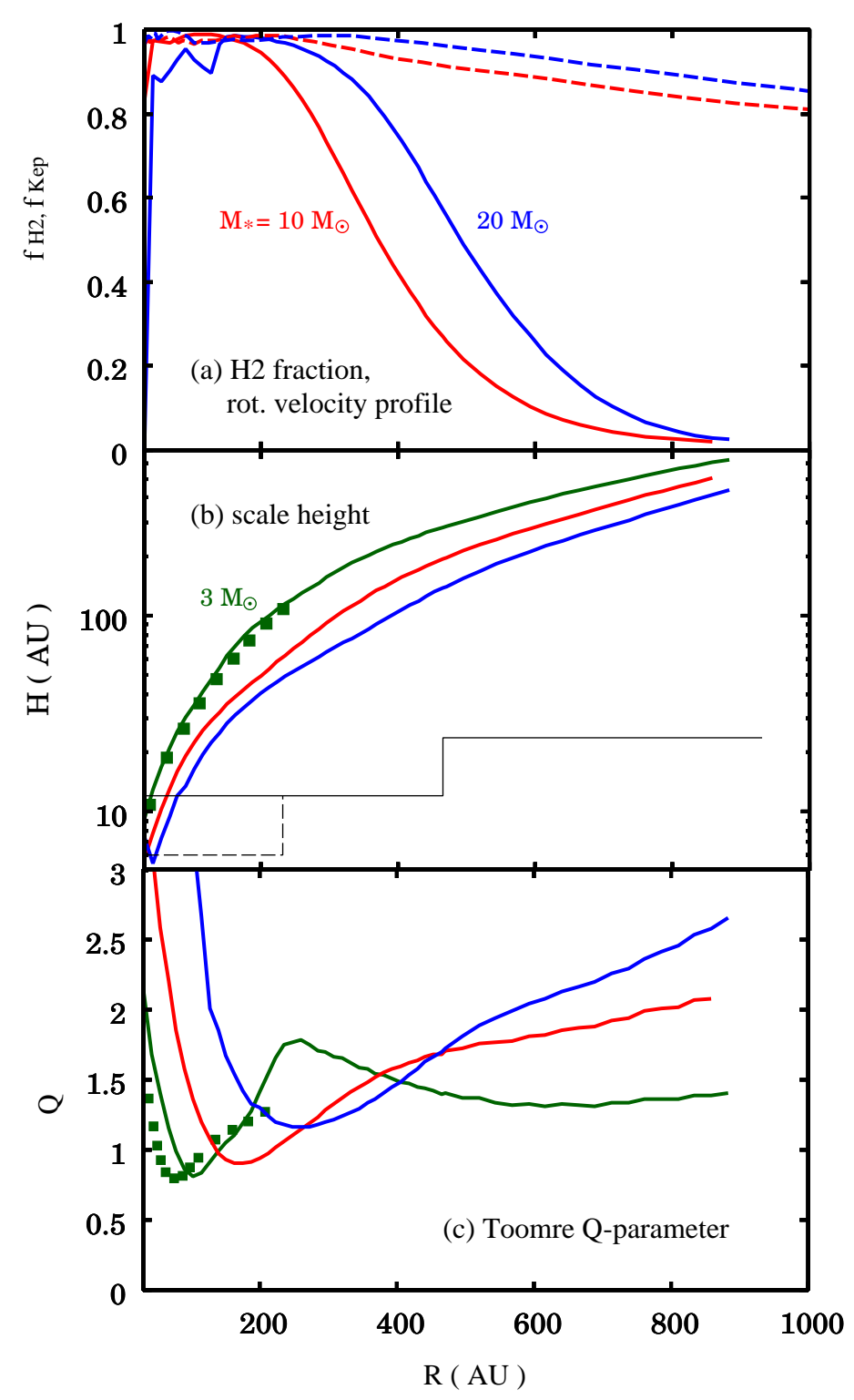

Fig. S5: Radial structure of the circumstellar disk within $1000 \mathrm{AU}$ around the protostar when the stellar mass is $3 M_{\odot}$ (green lines for the lower two panels), $10 M_{\odot}$ (red lines) and $20 M_{\odot}$ (blue lines). Upper panel: The fraction of hydrogen molecules along the equator $f_{\mathrm{H}_{2}}$ (solid lines), and the ratio of the local rotational velocity to Kepler velocity $f_{\text {Kep }}$ (dashed lines). Middle panel: The scale height of the disk $H$. The black thin line shows the grid resolution in our standard cases, and the discontinuity at $R \simeq 470 \mathrm{AU}$ indicates a grid-level boundary there. The profile when the stellar mass is $3 M_{\odot}$ with the doubled spatial resolution for $R<240 \mathrm{AU}$ is also presented (green filled squares). The black thin dashed line shows the grid resolution in this case. Lower panel: Same as the middle panel but for Toomre Q-parameter at each radius. 


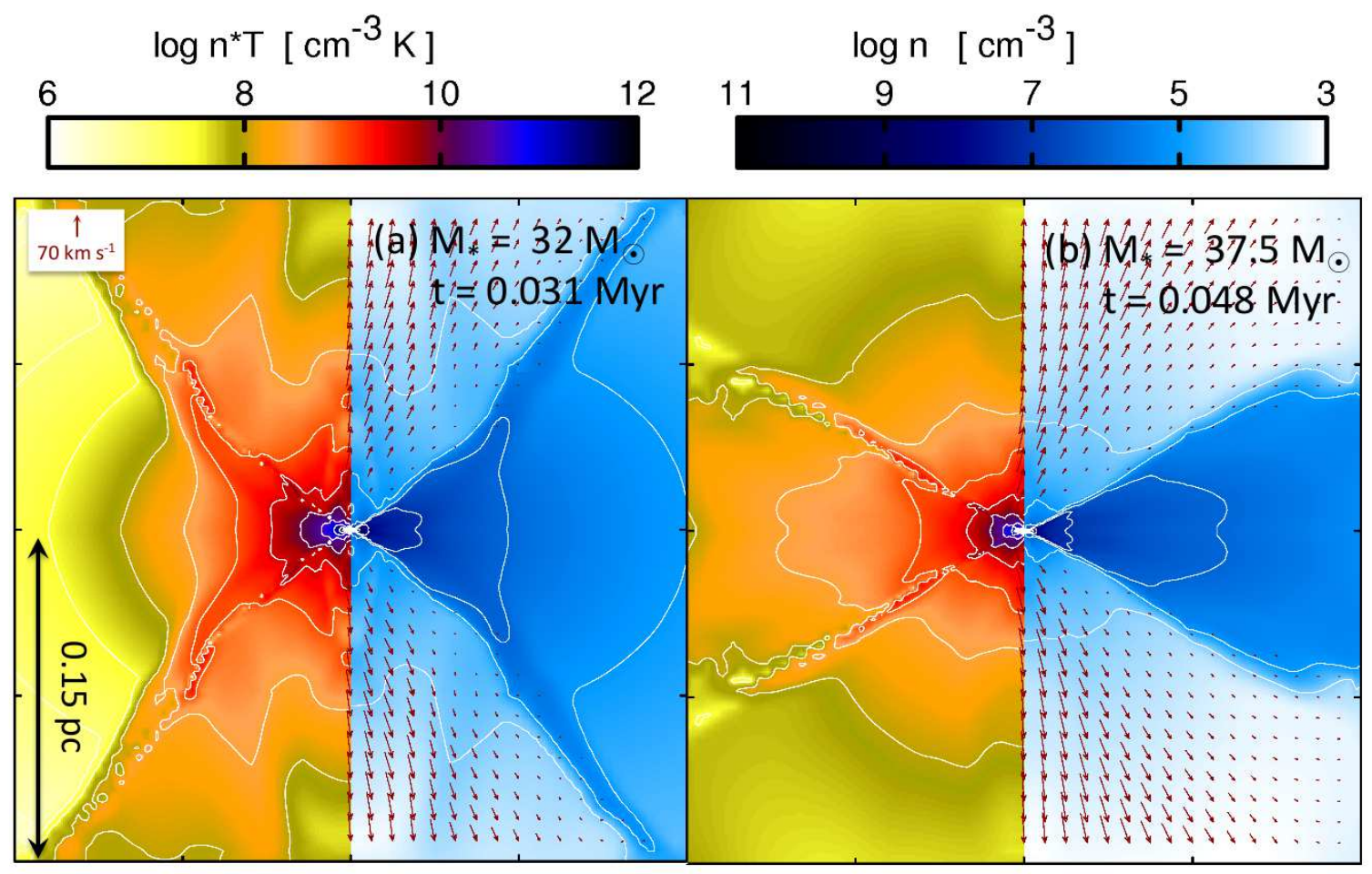

Fig. S6: Same as Fig. 2 in the main article but for gas temperature multiplied by number density in the left panel, which is nearly proportional to gas pressure. The left and right panels show snapshots at times, when the stellar mass is $M_{*}=32 M_{\odot}$ and $37.5 M_{\odot}$ respectively. 


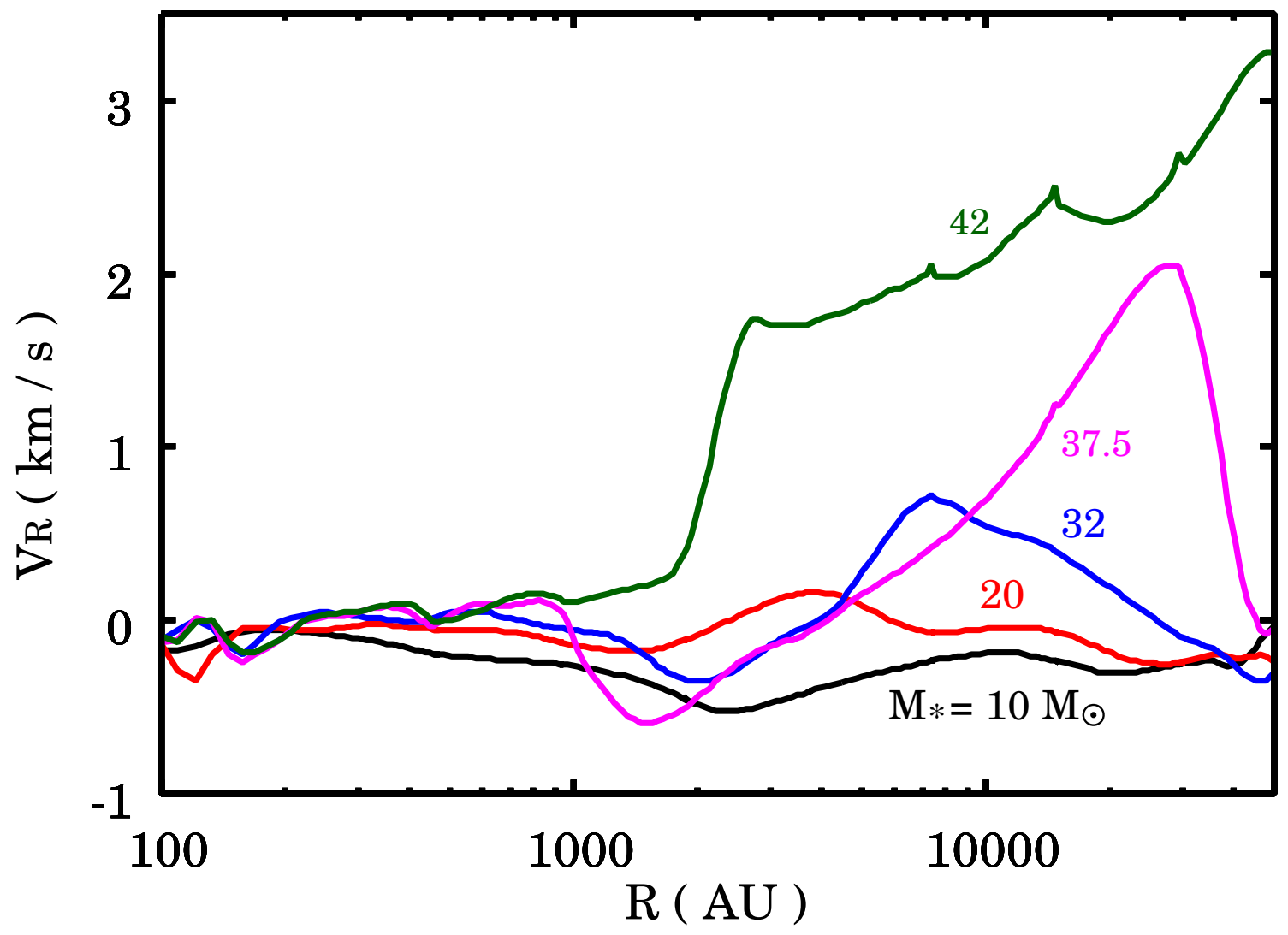

Fig. S7: Distribution of $R$-component of the radial velocity $v_{R}$ along the equator $(Z=0)$. The snapshots when the stellar mass is $10 M_{\odot}$ (black), $20 M_{\odot}$ (red), $32 M_{\odot}$ (blue), $37.5 M_{\odot}$ (magenta), and $42 M_{\odot}$ (green) are plotted. Positive $v_{R}$ means that gas is moving outward. 